Bulletin of the Natural History Museum, 2020, 13: 171-201.

Received 18 Mar 2020; Accepted 28 Oct 2020.

doi:10.5937/bnhmb2013171P

UDC: 069.6:582.082.5(497.113))

$581.92(497.113)$

Original scientific paper

\title{
MATERIALS FOR A FLORA OF SERBIA FROM THE HERBARIUM COLLECTION PZZP (3)
}

\author{
RANKO PERIĆ $^{1 *}$, JELENA KNEŽEVIĆ ${ }^{2}$ \\ ${ }^{1 *}$ Institute for Nature Conservation of the Vojvodina province, Radnička 20a, \\ 21000 Novi Sad, Serbia, e-mail: ranko.peric@pzzp.rs \\ ${ }^{2}$ Department of Biology and Ecology, Faculty of Sciences, University of Novi \\ Sad, Trg Dositeja Obradovića 2, 21000 Novi Sad, Serbia
}

This article presents the third part of data on selected new and noteworthy taxa ensuing from the revision of Herbarium collection of the Institute for Nature Conservation of the Vojvodina province (PZZP). Selected taxa include 11 species, three subspecies, three varieties, one infraspecific taxon with indetermined status [stat. indet.] and one nothospecies placed within seven genera (Filago L., Ludwigia L., Luzula DC., Lycopus L., Lythrum L., Phlomis L. and Silene L.). Two varieties (Luzula campestris subsp. campestris var. elata, Silene flos-cuculi subsp. floscuculi var. latifolia) and one infraspecific taxon with indetermined status (Luzula multiflora subsp. multiflora [stat. indet.] b. uliginosa) are new for a flora of Serbia. Additionally, one species (Ludwigia palustris) and one nothospecies (Lythrum

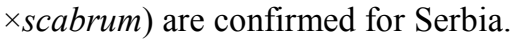

Key words: botanical collections, flora, chorology, Serbia

\section{INTRODUCTION}

In this, third installment of data resulting from our continuing work on the identification, revision and publishing of selected new and noteworthy taxa from the Herbarium collection of the Institute for Nature Conservation 
of the Vojvodina province (PZZP) (Perić et al. 2018; Perić \& Knežević 2019) are included data for genera Filago L., Ludwigia L., Luzula DC., Lycopus L., Lythrum L., Phlomis L. and Silene L.

\section{MATERIAL AND METHODS}

Material and methods are the same as in our previous contributions (Perić et al. 2018; Perić \& Knežević 2019): selection of vascular plant taxa (including nothotaxa) is based on the following criteria: 1) new (unpublished) taxa for a flora of Serbia, 2) otherwise known taxa documented so far with only a few published records for Serbia, 3) taxa with unclear or questionable distribution in Serbia due to recent substantial changes in their taxonomical concept, 4) revised or supplemented published data and 5) taxa protected by law in Serbia.

Unless otherwise stated, nomenclature follows the Euro+Med Plant Base (2006+) and "An annotated checklist of vascular flora of Serbia" (Niketić et al. 2018b). Author citations are given according to Rec. 46A, note 1 of the Code (Turland et al. 2018). When citing the types, the term "scan" means a HD picture available online through official herbarium websites or digital platforms (e.g. Herbarium WU, Herbarium of the Martin Luther University of Halle-Wittenberg). Distribution data are mapped on the $10 \times 10 \mathrm{~km}$ MGRS UTM maps (Lampinen 2001) within UTM Grid Zone 34T. Geographical regionalization of Serbia is cited according to Stevanović (1999). Abbreviations for herbarium collections used in the text are given according to Thiers (2016+).

\section{RESULTS AND DISCUSSION}

Filago minima (Sm.) Pers., Syn. Pl. 2: 422 (1807).

NeW DATA: SE Serbia: Pčinja: Kozjak Mt.: EM 78 Delinovica ["Delinovce"] (Perić, $R$. 26-Jul-2006).

PUBLISHED DATA: Šumadija: DQ 70 [subnom. Filago minima Fr.] Žabare, forest clearings (Pančić 1856: 550); DP 89 [subnom. Filago minima Fr.] Trnava, forest clearings (Pančić 1856: 550); DP 88 [subnom. Filago minima Fr.] Čumić, forest clearings (Pančić 1856: 550); DP 97 [subnom. Logfia minima (Sm.) Dumort.] Kragujevac ["Kragujevar"] ("Collib. Aspen. [asperis] ad urbical [urbicam] Kragujevar" [Kragujevac], 1858, Banien (P) Andrés-Sánchez et al. 2013: 15).

E Serbia: Vidlič Mt.: [subnom. Logfia minima (Sm.) Dumort.] FN 38 Mali vrh, $635 \mathrm{~m}$ and $620 \mathrm{~m}, \mathrm{~W}$ exposure (Marković et al. 2015: 64); FN 37 Vučje, 490 $\mathrm{m}, \mathrm{W}$ exposure (Marković et al. 2015: 64) $+650 \mathrm{~m}, \mathrm{~S}$ exposure, thermophilous oak 
forests and oriental hornbeam scrubwood (Marković et al. 2018: 106) $+1180 \mathrm{~m}$ (Jotić et al. 2011: 94), Tepoš plateau: Begova rudina, $651 \mathrm{~m}$ (Jotić et al. 2013: 22).

SE Serbia: EN 81 Vranjska Banja, sandy places [subnom. Filago minima Fr.] (Ničić 1893: 45).

S Serbia: EN 62 [subnom. Logfia minima (Sm.) Dumort.] Golemo Selo (Martínez-Ortega, M. M. et al. 21-Jul-2010, Andrés-Sánchez et al. 2013: 15); Rujan Mt.: EM 69 Košarno, “ass. Hyperico-Trifolietum trichopteri”, 1010 m, E, S exposure (Ranđelović \& Stamenković 1991: 30).

Kosovo: Podujevo: EN 24 Golak ["Golak Orlan”], "ass. Trifolio-Festucetum vallesiacae”, 1010 m, SW exposure (Ranđelović et al. 1979: 984).

IMPRECISE PUBLISHED DATA: Serbia [subnom. Filago minima Fr.; Filago montana L.; Filago montana f. montana] (Pančić 1874: 405; Hayek 1931: 593; Gajić 1975: 36; 1980a: 123); Fruška Gora Mt. ("dry, sunny habitats, rare" Obradović 1966: 108); Rogozna Mt. (Papović et al. 2014: 26); Vlasina [subnom. Logfia minima (Sm.) Dumort; Filago montana L. f. intermedia (Beckm.) Nyár.] (Košanin 1910a; Pančić, J. 1880, Jovanović, Niketić 1991 Ranđelović \& Zlatković 2010: 96; "meadow" Gajić 1973: 22; 1975: 36).

NoTES: Probably more widespread in parts of eastern, southeastern and southern Serbia (Fig. 1). Its only literature record for Vojvodina (Obradović 1966: 108) appear to be based on data published by Schulzer et al. (1866: 106) as "Filago montana L." for historic geographic area vaguely defined there as "Slavonia" which, according to this interpretation, includes parts of modern day Croatia and Serbia. This citation was later freely interpreted by Zorkóczy (1896: 80) as confined to Srem and furthermore restricted to Fruška Gora Mt. by Obradović (1966: 108). However, both claims remain unsupported with herbarium specimens. As a matter of fact, our herbarium and field data indicate that the only members of the genus Filago L. in Vojvodina are Filago germanica (L.) Huds. (very common in steppic and dry grassland habitats) and $F$. arvensis L. (less common, but known from Fruška Gora Mt., Vršac Mts. and Deliblato Sands).

CORRECTIONS: Herbarium specimens collected by N. Babić on Fruška Gora Mt. (Iriški Venac) and published under the name "Gnaphalium luteoalbum L." (Obradović 1966: 109) are in fact referring to Filago arvensis L. Also, specimens collected by T. Soška in Deliblato Sands (Flamunda) and published by Broz (1951: 324) as "Filago arvensis" are more precisely referring to $F$. arvensis [stat. indet.] a. gracillima Schur, Enum. pl. Transs. 318 (1866).

Ludwigia palustris (L.) Elliott, Sketch. Bot. S. Carolina 1: 211 (1817).

NEW DATA: Srem: CQ 68 Bosut forests: Obodnjača swamp, 44 $58^{\prime} 15.45^{\prime \prime}$ N, 019 $15^{\circ}$ 02.75” E, 86 m (Perić, R. 09-Nov-2017). 


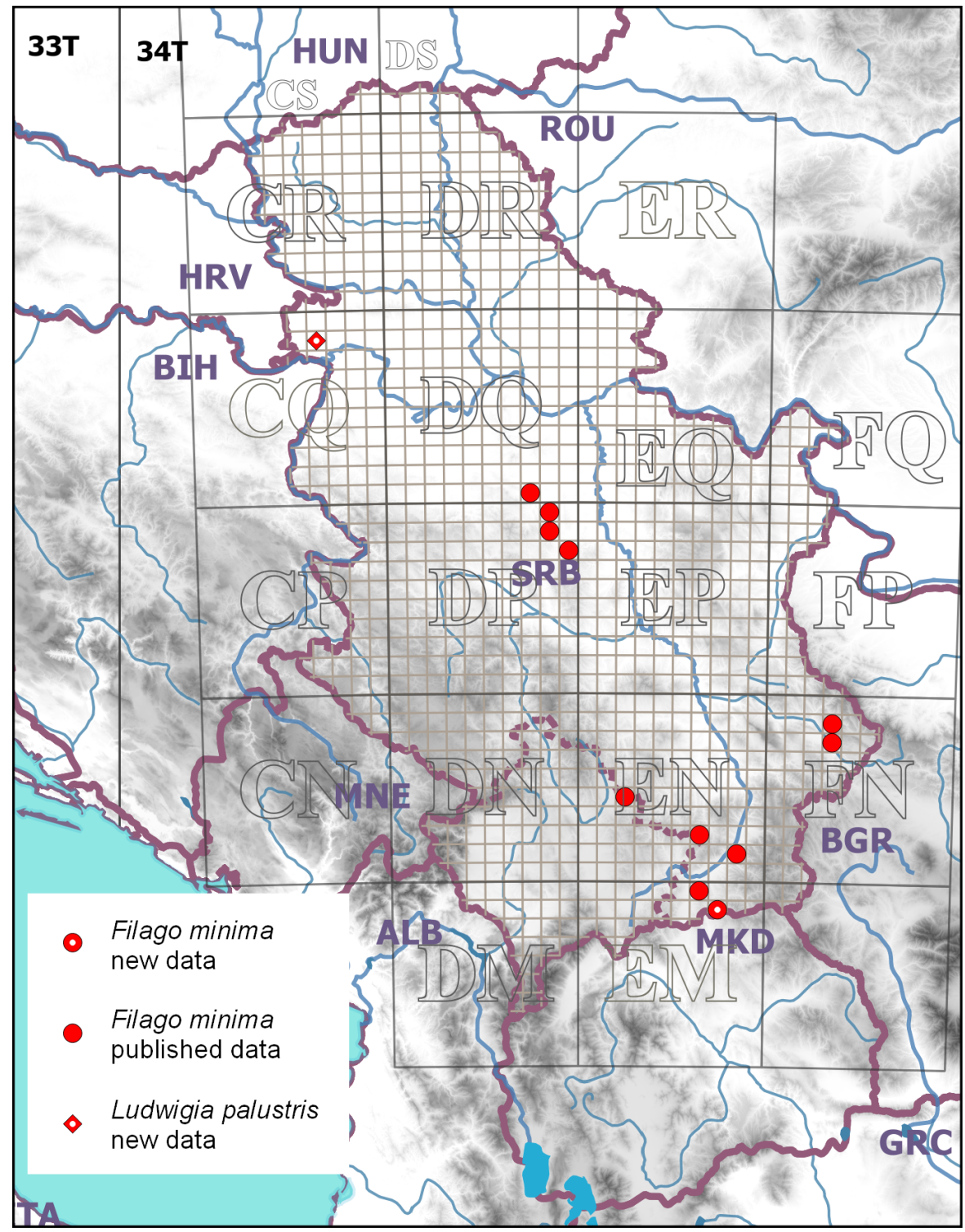

Fig. 1. - New herbarium and published data on the distribution of Filago minima (Sm.) Pers. and Ludwigia palustris (L.) Elliott in Serbia.

PUBLISHED DATA: Srem: Bosut forest area (Demeter et al. 2018: 118; Kiš et al. 2019; Biró et al. 2019; 2020).

NoTES: This regionally rare wetland species (Dítě et al. 2017: 35) has only recently been discovered for Serbia and published as present in large Bosut forest complex without reference to any precise location (Demeter et al. 2018; Kiš et al. 2019; Biró et al. 2019; 2020) (Fig. 1). We found it at 
forest pond Obodnjača swamp (Fig. 2) in a typical amphibious habitat developed on the muddy bottom and characterised with presence of amphibious and aquatic species like Agrostis stolonifera L., Callitriche palustris L., Chaiturus marrubiastrum (L.) Ehrh. ex Rchb., Eleocharis palustris (L.) R. Br., Hottonia palustris L. (2 individuals), Lindernia procumbens (Crock.) Philcox, Persicaria hydropiper (L.) Delarbre and Ranunculus trichophyllus Chaix. The population is a rather small, comprising about a hundred individuals unevenly dispersed on the area of a few square meters. According to Biró et al. (2019) traditional pig-herding practice, which is still present in Bosut forest area, has essential role in creating and preserving suitable habitats for Ludwigia palustris. The closest next records to this are known from the valley of the river Sava in Croatia and in south-southwestern Hungary [https://hirc.botanic.hr/fcd/] (Dítě et al. 2017).

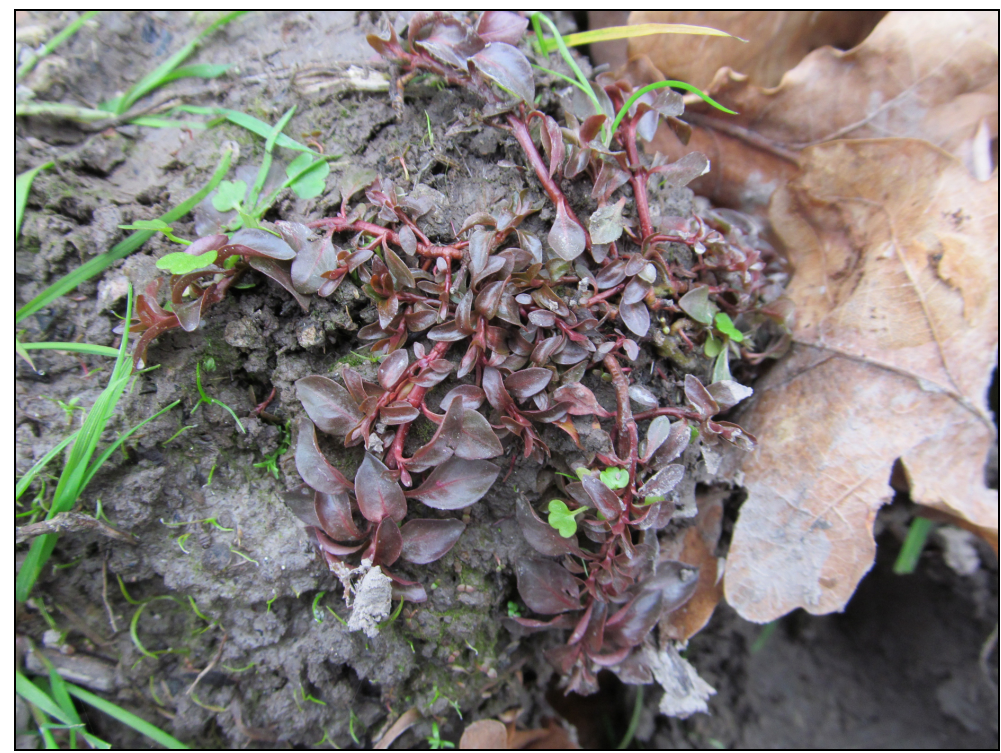

Fig. 2. - Ludwigia palustris (L.) Elliott, Obodnjača swamp (Bosut forests), 09. 11. 2017. (photo R. Perić).

Based on IUCN Red list categories and criteria (2012) its threat category in Serbia is critically endangered: CR B1a, b(i, ii, iii) $+2 a, b(i$, ii, iii), $\mathrm{C} 2 \mathrm{a}(\mathrm{ii})$.

Luzula campestris (L.) DC. in de Lamarck \& de Candolle, Fl. Franç., ed. 3,3: 161 (1805) [subsp. campestris] var. elata Schltdl., Fl. Berol. 1: 203 (1823) em. Soó, Syn. syst.-geob. fl. veg. Hung. 5: 130 (1973).

NeW DATA: E Serbia: Suva Planina Mt.: EN 98 Gornji Dušnik-Trem (Perić, R. 23-Jun-2005). 
NOTES: New for Serbia (Fig. 3). Vigorous plant up to $35 \mathrm{~cm}$ high with wider leaves, inflorescence composed of (3)4-6 flower clusters, each cluster comprising 8 to 10 flowers (Schlechtendal 1823: 203; Soó 1973: 130). Our specimens are highly comparable to type specimens deposited in the Herbarium of the Martin Luther University of Halle-Wittenberg (HAL 069139) [scan!] [https://herbarium.univie.ac.at/index.htm] (designated by Kirschner, 2002: 104).

Luzula exspectata Bačić \& Jogan, Taxon 56: 131 (2007).

NEW DATA: Metohija: Prokletije Mts.: Radavac: DN 43 Savine vode (Butorac, B. 30-Jun-1994); DN 32 Hasanov Vrh (Grubač, B. 17-Jul-1996) (Fig. 3).

PUBLISHED DATA: C Serbia: Kopaonik Mt.: DN 89 Suvo Rudište, northern slope, acidic meadow, 1750-1850 m (13-Jun-2006 Bačič et al. 2016: 59).

Kosovo: Šar-Planina Mt. ["Sharri Mts."]: EM 07-EM 17 Ljuboten ["Mt Luboten"] (Berisha et al. 2020: 129).

IMPRECISE PUBLISHED DATA: Serbia (Bačič et al. 2015: 48); Central Serbia (Niketić et al. 2018b: 89); Kosovo and Metohija (Niketić et al. 2018b: 89).

QUESTIONABLE PUBLISHED DATA [subnom. Luzula sudetica (Willd.) DC., "Luzula nigricans Desv.; Luzula congesta"]: C Serbia: Kopaonik Mt.: [subnom. Luzula sudetica (Willd.) DC.] DN 89 Krčmar (Nikolić 1976: 150).

E Serbia: Stara Planina Mt.: [subnom. Luzula congesta] FP 20 Dojčino Vrelo, "ass. Carici-Sphagno-Eriophoretum" "ass. Scirpetum silvatici", 1550 m, NE exposure, red sandstones and conglomerates (Mišić et al. 1978: Phyt. Tab. nos. 68, 69), "the lowest peatbog developed along the stream running from Dojčino vrelo", 1560 m (Jovanović-Dunjić 1971: 98); [subnom. Luzula congesta] FP 30 Babin zub, "ass. Scirpetum silvatici", $1550 \mathrm{~m}, \mathrm{NE}$ and NW exposure, red sandstones and conglomerates (Mišić et al. 1978: Phyt. Tab. no. 69); [subnom. Luzula congesta Lej., Luzula congesta (Thuill.) Lej.] FP 40 Martinova Čuka + Vražja Glava (Nikolić 1976: 148), mountain pastures (Adamović 1908: 193); [subnom. Luzula congesta Lej., Luzula congesta (Thuill.) Lej.] FN 49 Kopren (Nikolić 1976: 148), mountain pastures (Adamović 1908: 193); [subnom. Luzula congesta] FN 48 Ponor-Lokve, "ass. Caricetum goodenowii", $1350 \mathrm{~m}$, SW exposure, red sandstones and conglomerates (Mišić et al. 1978: Phyt. Tab. no. 67).

Metohija: Prokletije Mts.: [subnom. Luzula sudetica (Willd.) DC.] DN 22 Nedžinat, "ass. Pinetum mughi-Geum bulgaricum", 2100-2120 m, NE exposure, carbonate (Amidžić 1997: 92; Amidžić \& Krivošej 1998); [subnom. Luzula sudetica (Willd.) DC.] DN 32 Lumbardska Planina (Nikolić 1976: 150); [subnom. Luzula sudetica (Willd.) DC.] DN 31-DN 32 Koprivnik (Grebenščikov 1939, Grebenščikov 1943; Diklić \& Nikolić 1961: 231; Nikolić 1976: 150); DN 21 [subnom. Luzula sudetica (Wild.) DC.] Bogdaš ["Marjaš"], "ass. Elyno-Edraianthetum alpini", $2300 \mathrm{~m}, \mathrm{~N}-\mathrm{NW}$ exposure, slope 20², rendzina (31-Aug-1967, Lakušić 1968: Phyt. tab. no. 14); Derviš Kom (Černjavski, Rudski, Lindtner 1933, Diklić \& Nikolić 1961: 231); Kurvala (Nikolić 1976: 150); [subnom. Luzula sudetica (Willd.) DC.] DN 20 Djeravica ["Djaravica"] (Nikolić 1976: 150; Amidžić \& 
Krivošej 1996: 30), "ass. Saxifragetum cymosae", 2400 m, W exposure, silicate (Amidžić \& Stevanović 1996: 35); Šar-Planina Mt.: [subnom. Luzula sudetica (Willd.) DC.] DM 74 Šutman (Randjelović et al. 1998: 380), Šutman plateau (Amidžić et al. 1999: 65); [subnom. Luzula congesta] DM 97 Ostrovica, "ass. Pinetum heldreichii peuces scardicum", 1700-1730 m, northern and northeastern serpentine slopes, slope $20^{\circ}-30^{\circ}$, shallow lithosoil (Stevanović 1995: 94).

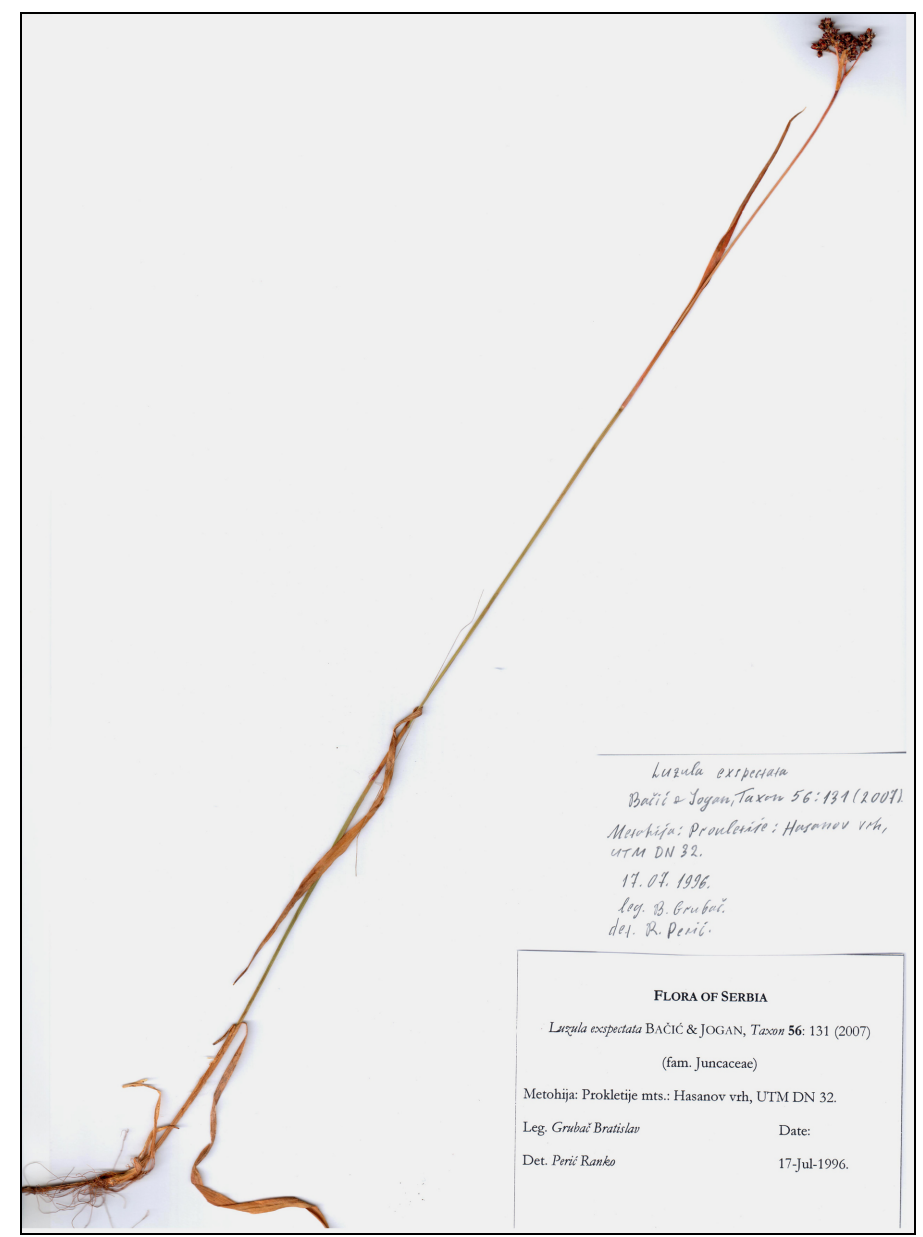

Fig. 3. - Luzula exspectata Bačić \& Jogan (Prokletije Mts, Radavac, Hasanov Vrh, PZZP).

Kosovo: Šar-Planina Mt.: ["Scardus"] [subnom. Luzula nigricans Desv.; Luzula sudetica (Willd.) DC. in Lam.] DM 86-DM 96 Kobilica ["Kobelitza"] ("sparsely in meadows of alpine region" "alt. 5-7000" [1500-2100 m]" "mica schist substrate", Grisebach 1844: 405; Duraki et al. 2017: 8, 15).

QUESTIONABLE \& IMPRECISE PUBLISHED DATA: Serbia [subnom. Luzula sudetica; Luzula congesta (Thuill.) Lej.] [“Serbien”] (Adamović 1899: 379; Gajić 1980a: 128); Eastern Serbia [“Ostserbien"] [subnom. Luzula congesta], alpine- 
mats (Adamović 1898: 189); Željin Mt. [subnom. Luzula nigricans Desv.] (Pančić 1856: 582); Kopaonik Mt. [subnom. Luzula sudetica (Willd.) DC.] (Gajić et al. 1991: 655; Lakušić 1996: 30); Stara Planina Mt. [subnom. Luzula multiflora (Ehrh.) Lej. subsp. congesta (Thuill.) Arcangeli] (Ivančević et al. 2007: 197); Šar Planina Mt. [subnom. Luzula sudetica] (Amidžić \& Ostojić 2006: 41).

NOTES:

1. This high-mountain species, which was described only recently, had been treated long by botanists as either L. sudetica (Willd.) Schult. or some form of L. multiflora with crowded flowers (Bačič et al. 2016: 55). Compared with L. sudetica it has clearly developed and longer caruncle $(0.2-0.8 \mathrm{~mm}$ long $v s$. up to $0.1 \mathrm{~mm}$ long, rather filiform caruncle in L. sudetica), larger all flower and fruit parts (cf. Bačič et al. 2007: 132; Fig. 4d, Bačič et al. 2016: 56) and Balkan distribution ( $L$. sudetica is absent from the Balkans). From forms of L. multiflora subsp. multiflora with crowded flowers (see in the next section) it principally differs by \pm unequal outer and inner tepals (at least in some flowers outer tepals are clearly longer than inner tepals vs. equal outer and inner tepals in L. multiflora) and by elliptic, greenish or pale brown seeds with smaller caruncle ( $v s$. more globular, dark green seeds with large caruncle, about $1 / 3$ up to $1 / 2$ as long as the rest of the seed in multiflora) ( $c f$. Fig. 4c. in Bačič et al. 2016: 56). Its natural range is a rather disjunct with majority of the confirmed records limited to the southeastern Alps (in Slovenia and adjacent parts of Austria and Italy) and with isolated records from some high-mountains in Serbia and North Macedonia (Šar Planina Mt., Kopaonik Mt., Pelister Mt.) (Fig. 4). As a member of one of the most intricate taxonomic group in Europe (Luzula sect. Luzula) with numerous instances of literature data plagued by examples of misidentification or confusion with other similar species within the group, even in the countries with long and continuous botanical tradition, published data pertaining to distribution of this species in botanically less-explored areas such as the Balkans (Bačič et al. 2016: 52) should be treated at least with caution. Also, our field observations and herbarium specimens suggest that data for $L$. congesta published for eastern Serbia are to a certain extent possibly related to one slender mountain form of $\boldsymbol{L}$. multiflora with crowded blackish flowers ([stat. indet.] b. uliginosa) which appears to be particularly common in high-mountain habitats of Stara Planina Mt. (see next section). According to Niketić et al. (2018b: 89) L. multiflora 
subsp. congesta does not occur in Serbia (it is typical for western atlantic Europe and Scandinavia, Kirschner 2002: 108).

2. Records of L. sudetica published by Rechinger (1935:379) for Prokletije Mts. (Lumbardska Planina Mt., Devojački Krš, Čakor, Kurvala, Djeravica) are later revised as L. multiflora (Rechinger 1952:338).

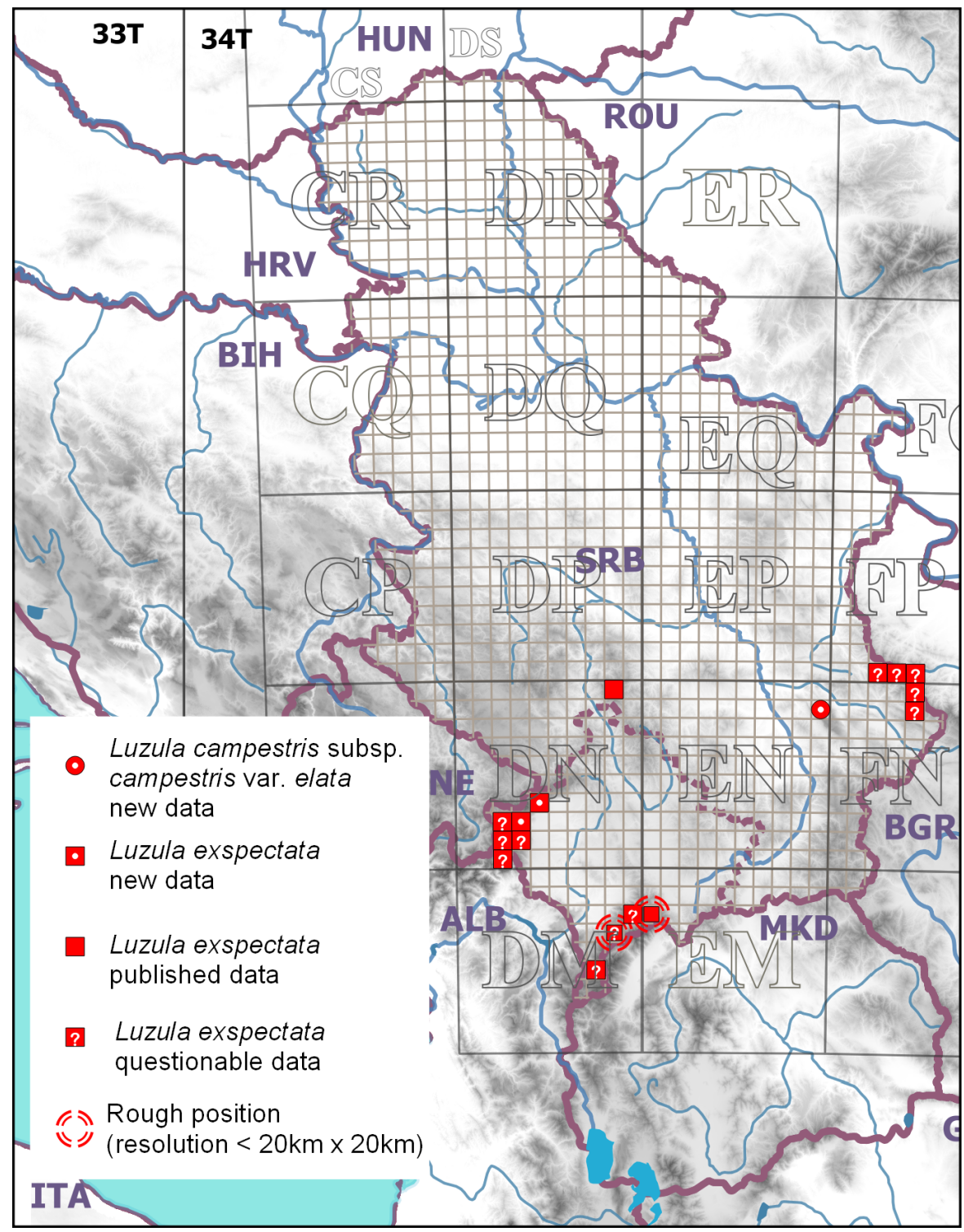

Fig. 4. - New herbarium and published data on the distribution of Luzula campestris [subsp. campestris] var. elata Schltdl. and L. exspectata Bačić \& Jogan in Serbia. 
Luzula multiflora (Ehrh.) Lej., Fl. Spa 1: 169 (1811) [subsp. multiflora] [stat. indet.] b. uliginosa Gremli, Excursionfl. Schweiz, ed. 3, 377 (1878).

NEW DATA: E Serbia: Suva Planina Mt.: EN 98 Devojački Grob-Trem (Perić, R. 23-Jun-2005); Stara Planina Mt.: [subnom. Luzula] FP 30 Midžor (Perić, R. 02-Aug-2010).

Metohija: Prokletije Mts.: DN 33 Štedim, meadow (Panjković, B. 17-Jul-1996).

NOTES: New for Serbia (Fig. 5). While typical L. multiflora subsp. multiflora mostly occurs in humid grassland and woodland habitats, our specimens are indicating that in somewhat more arid habitat types are present two less typical, \pm slender or dwarf variants of $L$. multiflora with crowded and smaller inflorescences comprising fewer, shortly pedunculate or (sub)sessile flower clusters.

1. [stat. indet.] b. uliginosa Gremli is a common form of highmountain grasslands and pastures. It was described from Switzerland and has a several slender stems with 2-6 shortly stalked or almost sessile flower clusters with perianth segments and capsules deeply suffused with dark-blackish tinge (Gremli 1878: 377; Kirschner 2002: 110). It is somewhat similar to L. exspectata from which clearly differs by perianth segments, capsule size, seed and caruncle characters (see note 1 in the previous section). Part of data published for Stara Planina Mt. under the name "Luzula congesta" possibly refer to this taxon. Also recorded in Bosnia \& Herzegovina (Zelengora Mt.: vicinity of Borovno brdo: Hill 1813, Perić, R., Škondrić, S. 16-Aug-2011, PZZP) and Montenegro (Durmitor Mt.: Barno jezero, Perić, $R$. Jul-2005).

2. The second variant confined to steppic and sand-steppic habitats in Vojvodina was described under the names $L$. intermedia Figert, Deutsche Bot. Monatsschr. 15: 12 (1897), nom. illeg. and $\boldsymbol{L}$. ambigua Soó, Acta Bot. Acad. Sci. Hung. 16: 367 (1970), pro hybr. It has few shortly pedunculate or subsessile, densely-flowered clusters (sometimes on slightly curved pedicels as in L. campestris), anther-filament ratio of 1-2 and large caruncula (about $1 / 2$ of seed lenght), which induced some botanists to present it as a hybrid between L. multiflora subsp. multiflora and L. campestris subsp. campestris (Soó 1970b: 367). This claim was not confirmed neither by karyological examination nor by experimental hybridization (Kirschner 1991: 83). According to Kirschner (2002: 110 ) it represents synonym of L. multiflora subsp. multiflora. It differs from [stat. indet.] uliginosa by its dwarf growth, antherfilament ratio of 1-2 and light-brown to brown colour of perianth and capsules ( $v s$. in uliginosa plant stature and anther-filament ratio 
are as in typical multiflora and perianth and capsules have blackishdark brown colour). From L. fallax differs by glabrous and irregularly or sparsely scattered papillae along the whole lenght of the flower peduncles ( $v s$. flowering peduncles regularly and densely papillose in the upper part in fallax), richer flower clusters (composed of 8-18 flowers vs. 2-8 flowers in fallax) and longer

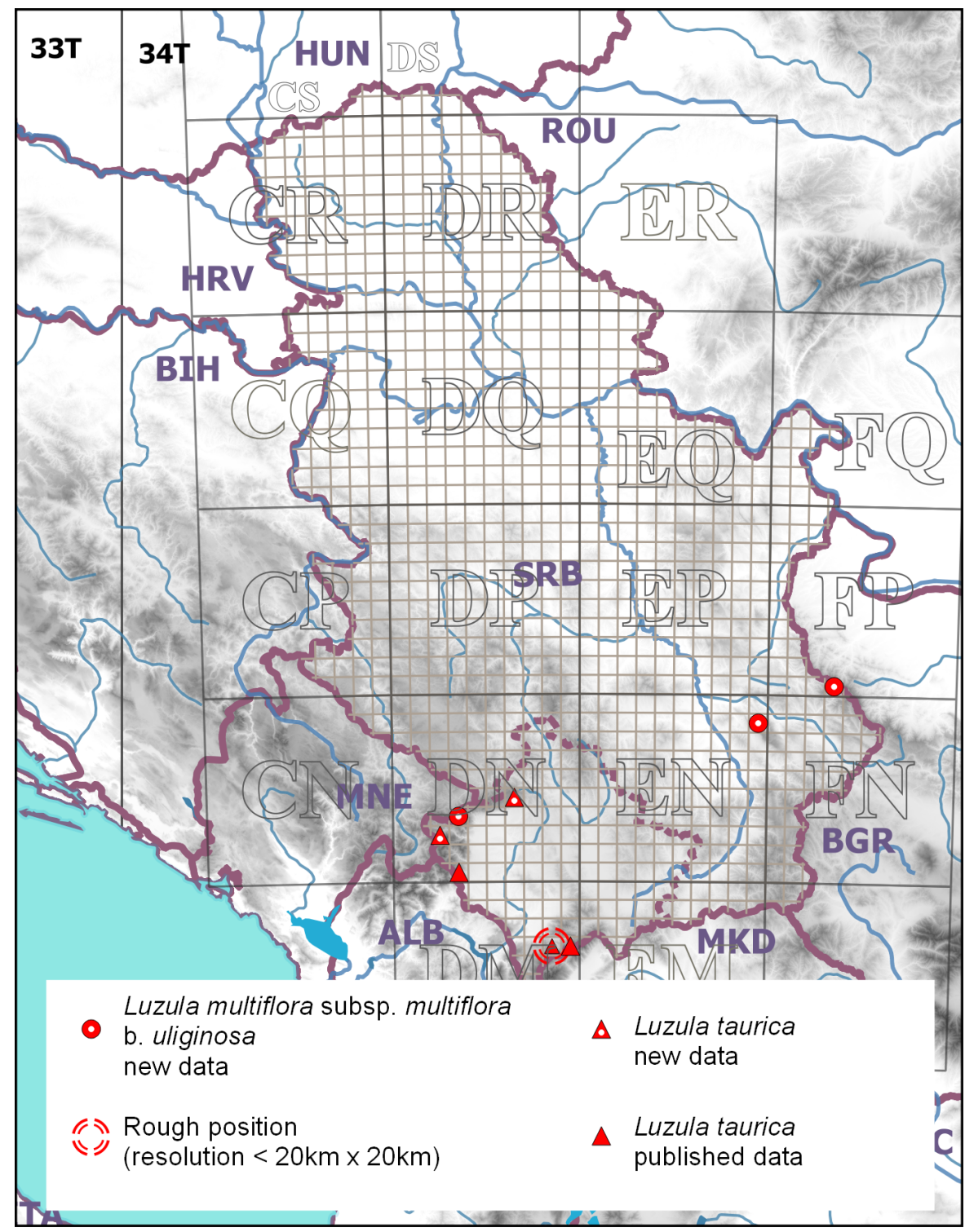

Fig. 5. - New herbarium and published data on the distribution of Luzula multiflora [subsp. multiflora] [stat. indet.] b. uliginosa Gremli and L. taurica (V. I. Krecz.) Novikov in Serbia. 
segments of capsula (ca. $3 \mathrm{~mm}$ vs. 2-2.5 mm in fallax) (Kirschner 2002; Bačič et al. 2016: 60; 2019: 153). Recorded in Fruška Gora Mt. (Vrdnik, humid meadows, forest edges, 17-May-2004, Galamboš, L.), vicinity of Banatska Palanka (Djavolji most, vicinity: near Hill 108.6, 08-May-2008, Perić, R.) and possibly in Deliblato Sands ("L. multiflora Lejeun.-Similis L. campestri Lam." Rochel 1838: 63).

Luzula taurica (V. I. Krecz.) Novikov, Nov. Sist. Vyssh. Rast. 27: 20 (1990).

NEW DATA: Metohija: Prokletije Mts.: Mokra Gora Mt.: DN $64 \underline{\text { Radopolje }}$ + Jerebinje (Panjković, B. 05-Jun-1996); DN 22 Rugovo Gorge, $1350 \mathrm{~m}$ (Panjković, B. 31-May-1996), ["Rugovo"] hay meadow in beech forest zone (Panjković, B. [s. dat.]).

Published Data: Metohija: Prokletije Mts.: DN 30 Djeravica: Mala Djeravica, eastern ridge, $2211 \mathrm{~m}$, relatively exposed alpine meadows with short snow cover over siliceous bedrock, $20.1572^{\circ}$ E, 42. $527^{\circ} \mathrm{N}$ (14-Aug-2010, Bačič et al. 2016: 59); Kosovo: ŠarPlanina Mt.: ["Shar Planina"]: DM 86-DM 96 Kobilica, $2520 \mathrm{~m}$ (Pawlowski, B. 27Aug-1938, KRAM, Kirschner 2002: 102); [subnom. Luzula atrofusca] DM 96 Bistra, "ass. Sesleria korabensis-Juncus trifidus", "ass. Carex curvulaSesleria comosa" (25-Jul, 28Jul, Rajevski 1990: 47, 49).

IMPRECISE PUBLISHED DATA: Kopaonik Mt. [subnom. Luzula campestris (L.) Lam., DC. 1805 subsp. alpestris (R. Bey) Kož. 1964 var. debilis (Vel.) Kož. 1964] (Lakušić 1996: 30); Kosovo and Metohija (Niketić et al. 2018b: 91).

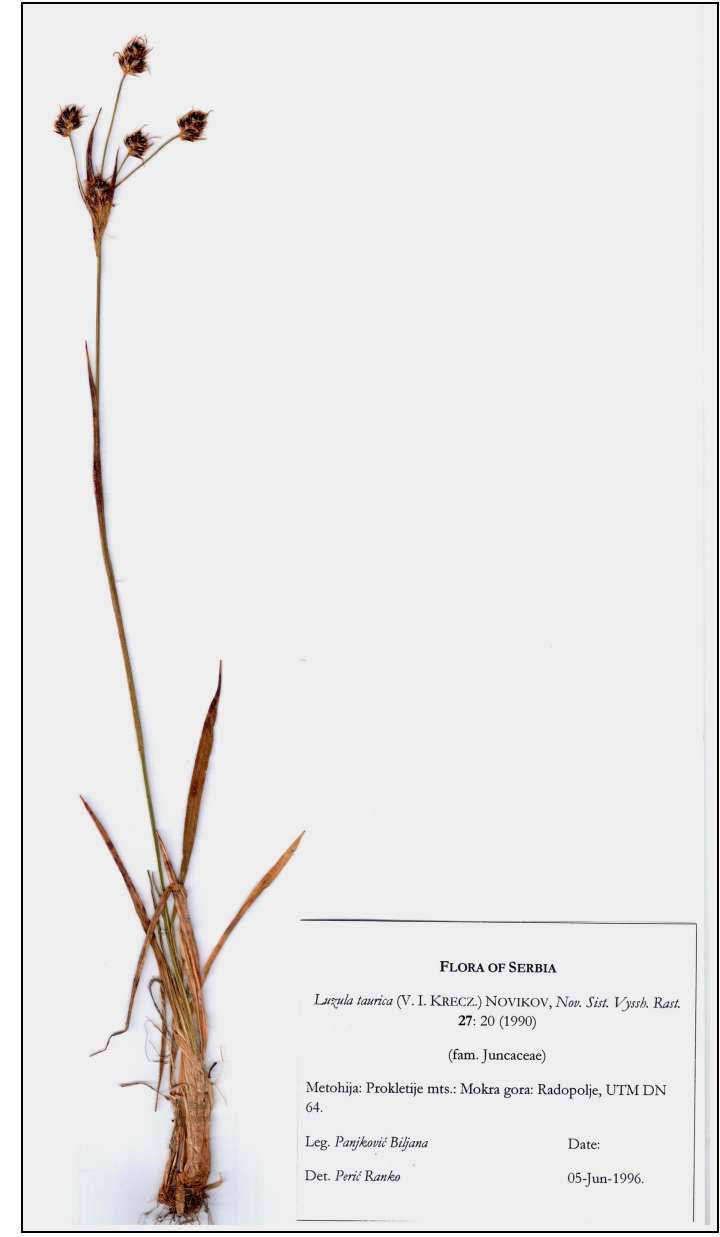

Fig. 6. - Luzula taurica (V. I. Krecz.) Novikov (Prokletije Mts.: Mokra Gora Mt.: Radopolje). 
NOTES: Similar to L. multiflora subsp. multiflora, but its inflorescence comprises 1 subsessile and 2-6 pedunculate clusters (vs. 3-10(12) in multiflora), perianth segments are almost always dark to blackish brown (vs. pale greenish, brownish, reddish to dark brown in multiflora), capsule shorter than perianth (vs. mostly as long as perianth in multiflora), stigma strikingly long $(0.7-1 \mathrm{~mm}$ long vs. $0.4-0.9 \mathrm{~mm}$ in multiflora), fruiting capsules ending with distinctly elongated persistent, spreading stigmas which give bristly outlook to flower clusters ( $v s$. stigma falling off in multiflora). High-montane meadows and pastures (Fig. 5). According to Bačič et al. $(2016: 56,59)$ this is likely to be one of the most common (sub)alpine species from sect. Luzula on the Balkan Peninsula (Prenj Mt., Čvrsnica Mt., Maglić Mt., Durmitor Mt., Komovi Mts., Prokletije Mts., Osogovske Mt.). Our specimens for the most part match type specimens of Luzula campestris var. atrofusca Maly, Glasn. Zemaljsk. Muz. Bosni Hercegovini 32: 141 (1920) (= L. taurica) deposited at the Herbarium collection of the Institute of Botany in Vienna (WU 033991) [scan!] [https://herbarium.univie.ac.at/database/detail.php?ID=103134] (Fig. 6).

ADDITIONAL CORRECTIONS FOR LUZULA: Herbarium specimens collected by N. Babić on Fruška Gora Mt. (Testera) and published under the name "Luzula pilosa (L.) Willd." (Obradović 1966: 150) are referring to Luzula luzuloides (Lam.) Dandy \& Wilmott subsp. luzuloides.

Lycopus europaeus L., Sp. Pl. 21 (1753) var. mollis (A. Kern.) Briq., Lab. Alp. Marit. 1: 117 (1891).

NEW DATA: Bačka: CS 91-DS 01 Subotica-Horgoš Sands ["Subotičke šume"] (Butorac, B. 18/22-May-1998).

PUBLISHED DATA: E Serbia: [subnom. Lycopus mollis Kerner] FP 02 Knjaževac: vicinity, wetlands (Adamović Fritsch 1918: 266).

NOTES: Stem densely arachnoid-lanate, leaves with long soft hairs beneath ( $v s$. stem and leaves with short hairs, subglabrous or glabrous in var. europaeus) (Kerner 1866: 371; Soó, 1968: 126-127). Possibly more widespread in Serbia (cf. "Lycopus europaeus L. (villosus) herb." Rochel 1838: 63) (Fig. 7).

Lythrum portula (L.) D. A. Webb, Feddes. Repert. 74: 13 (1967).

NEW DATA: Bačka: CR 45 Apatin: Kurjačica, forest puddle (Perić, R., 21May-2005, pers. comm.); CR 45 Apatin-Svilojevo: Slatina, pasture pond (Perić, R., 03-May-2006, pers. comm.); CR 53 Deronje: Osnovna bara swamp,

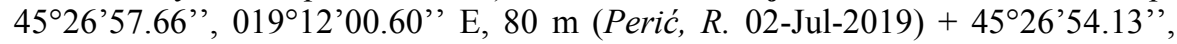
01912'11.45” E, 80 m (Perić, R., 10-Jul-2019, pers. comm.).

Banat: Novi Kneževac: DR 39 Filić: saline pasture adjacent to horse racecourse, in ditch (Perić, R. 22-Jun-2011); Jazovo: DR 48 Ladoman-Ištvanov 
salaš-Kasalo (Perić, R. 20-Aug-2009); DR 82 Sečanj-Boka: Čot, $\approx 45^{\circ} 21^{\prime} 03.37^{\prime \prime}$, 02047'17.94' E, 73 m (Perić, R., 29-May-2013, pers. comm.); Tomaševac: DR 61 Slatina, $\approx 45^{\circ} 15^{\prime} 11.17^{\prime}$ N, 020 37'00.54" E, $71 \mathrm{~m}$ (Perić, R., Stojšić, V. 01Jul-2011); DR 50 Farkaždin: Slatine, $\approx 45^{\circ} 10^{\prime} 24.05^{\prime}$, , 020²7’30.85' E, $68 \mathrm{~m}$ (Perić, R., 10-May-2013, pers. comm.); DR 50 Idvor, pasture, $\approx 45^{\circ} 11^{\prime} 08.49^{\prime \prime} \mathrm{N}$, 02029'14.42”' E, 71 m (Perić, R. 27-May-2011).

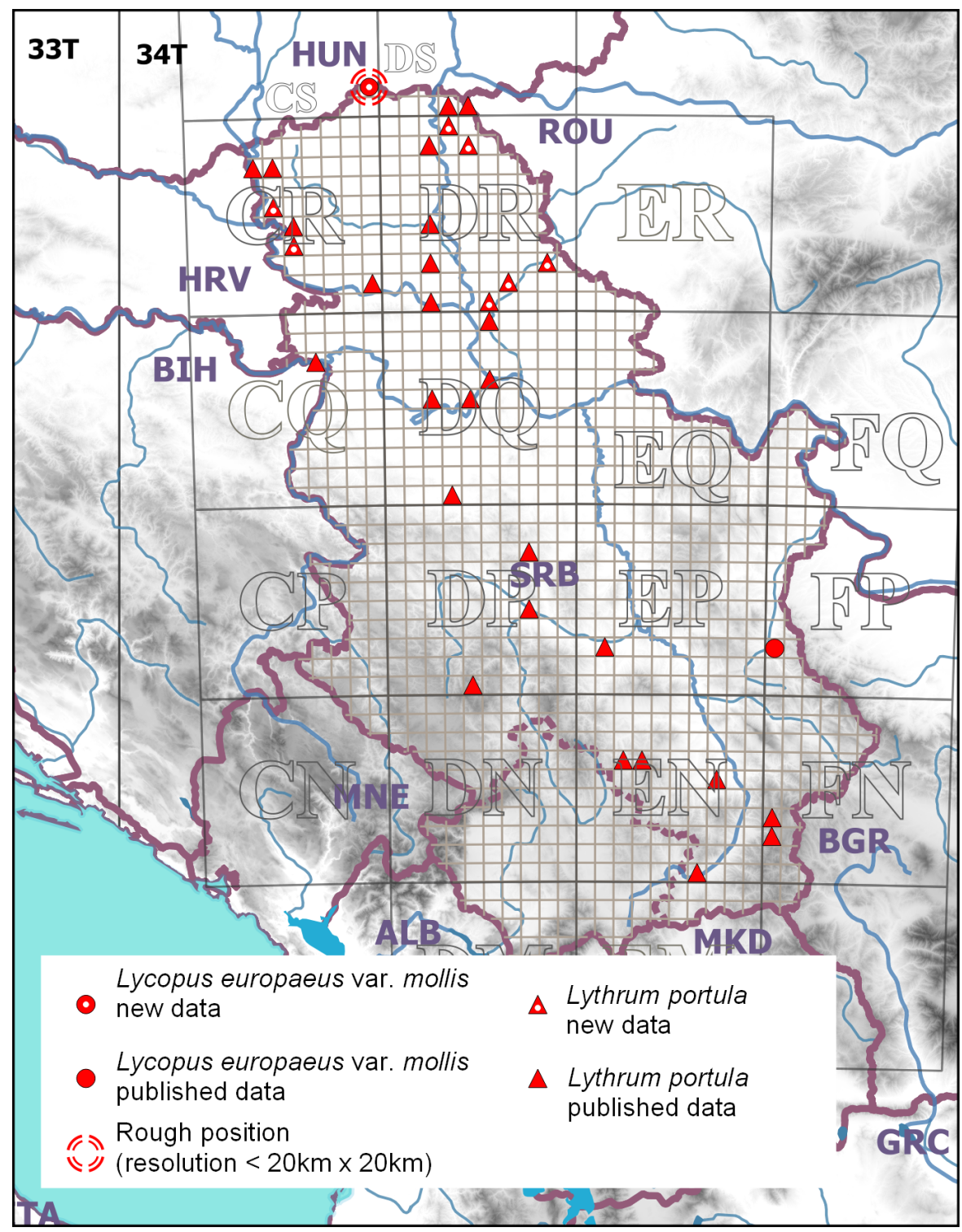

Fig. 7. - New herbarium and published data on the distribution of Lycopus europaeus var. mollis (A. Kern.) Briq. and Lythrum portula in Serbia. 
PUBLISHED DATA: Bačka: [subnom. Peplis portula L.] CR 37 Bački Monoštor (Budak 1998: 105); [subnom. Peplis portula L.] CR 47 Bezdan ["Bezdán"]: dried saline puddles (Prodán 1915: 242), Štrbac-Kozara forest ["Bezdáni erdő"], on wet and saline tussocks (Prodán 1914: 130), floodplain places (Prodán 1911: 328); [subnom. Peplis Portula L.] DR 28 Senta ["Zenta"]: Kaločka dolina ["Kalocsai völgy"], channel (Guelmino 1968: 43, 126); [subnom. Peplis portula L.] CR 54 Srpski Miletić (Budak 1998: 105); [subnom. Peplis portula L.] DR 24 Stari Bečej ["Óbecse"]: Donji rit ["Alsórét"], "quite widespread in swampy places and wet ditches" (1907 Kovács 1929: 128); Great Bačka Canal ["Ferencsatorna"] ("quite common" Kovács 1915: 74); [subnom. Peplis portula L.] DR 22 Žabalj (Djurčjanski 1980 Budak 1998: 105); [subnom. Peplis portula S.] CR 91 Futog ["Futtak"], forests in the vicinity ["futtaki- és felső erdő"], wetlands (Zorkóczy 1896: 26); [subnom. Peplis portula L.] DR 20 Kovilj (Budak 1978 Budak 1998: 105).

Banat: [subnom. Peplis portula L.] Siget: DS 30 Veliki Siget (Knežević 1994: 75); DS 40 Mali Siget (Knežević 1994: 75); [subnom. Peplis portula L.] DQ 59 Čenta (Knežević 1994: 75).

Srem: Srem: [subnom. Peplis portula L.] DQ 25 Obrež-Ašanja-Kupinovo: Matijevica forest [“Matijevica I", “Matijevica III"], (Slavnić 1950-1952: 164); CQ 67 Višnjićevo: Vinična: on the road between the forest compartments No. 37 and

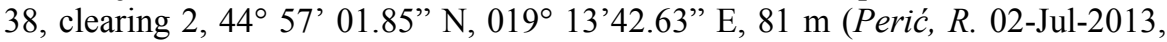
PZZP, Perić et al. 2016: 85); [subnom. Peplis portula L.] DQ 45 Jakovo (Moesz 1916: 2); [subnom. Peplis Portula L.] DQ 56 Zemun [“Semlin"] (Pančić Schulzer et al. 1866: 157; Schloser \& Vukotinović 1869: 307).

NW Serbia: Lajkovac: [subnom. Peplis portula L.] DQ 30 Bogovadja monastery (Černjavski, P. Gajić 1965: 33; Jovanović-Dunjić 1973: 8).

Šumadija: Kragujevac: [subnom. Peplis portula L.] DP 77 Ljuljaci, mountain puddles (Pančić 1856: 490); [subnom. Peplis portula L.] DP 74 Kraljevo (Jovanović-Dunjić 1973: 8).

W Serbia: Golija Mt.: [subnom. Peplis portula L. + f. callitrichoides] DP 40 Dajićko Lake (Gajić 1989: 250) + northwestern corner of the lake (Košanin 1908).

C Serbia: Kruševac: EP 12 Bela Voda-Kukljin-Bresno Polje-Globoder: Osredak (Stojković 2012: 70).

S Serbia: Sokolovica Mt.: EN 26 Presla summit, 800 m, "Querco-Carpinetum" (Tomović et al. 2005: 32); EN 36 Krompirište summit (Tomović et al. 2005: 32); Leskovac: [subnom. Peplis portula L.] EN 75 Donja Jajina [“Jajna”], near lake (Ilić, J. Formánek 1895: 345); [subnom. Peplis portula L.] EN 60 Bujanovac (Jovanović-Dunjić 1973: 8).

SE Serbia: Vlasina: [subnom. Peplis portula L.] FN 03 Gadžina bistrica + Čavdarova Reka (Košanin 1910a); FN 02 Bratanov del, mixed Sphagnetum (Katić 1910: 23).

IMPRECISE PUBLISHED DATA: Srbija [subnom. Peplis portula L.] (Pančić 1874: 303; Hayek 1926: 940; Domac 1950: 249; Čanak et al. 1979: 26; Gajić 1980a: 130; Janković 1985: 155); Vojvodina [subnom. Peplis portula L.], "ass. 
Elatine hungarica-Ammania verticillata", "ass. Lythrum tribracteatum-Lythrum hyssopifolia" (Slavnić 1951: 154, 156); Subotica Sands [subnom. Peplis portula L.] (Obradović \& Boža 1986: 132); North Banat [subnom. Peplis portula L.], "Nanocyperion flavescentis Koch 1926" (Adamović 1959: 36); West Bačka [subnom. Pepelis portula L.] (Grdinić et al. 2001: 148); Fruška Gora Mt.: [subnom. Peplis portula L.] along the river Danube, in flooded places, scattered (Obradović 1966: 49); Novi Sad [subnom. Peplis portula L.]: vicinity, along the Danube river (Jovanović-Dunjić 1973: 8); Belgrade [subnom. Peplis portula L.], vicinity (Černjavski 1950: 119; Janković 1972: 164); Šumadija [subnom. Peplis portula L.] (Gajić 1967: 190); Peštersko polje [subnom. Peplis portula L.] (Lazarević 2014: 13); Vlasina [subnom. Peplis portula L.] (Jovanović-Dunjić 1973: 8); Vlasina Lake [subnom. Peplis portula L.] along the stream (Košanin 1910b: 183), on barren peat islands, "ass. Bidentetum minimae" (Randjelović, $V$. 28-Aug-1992, Randjelović \& Zlatković 2010: 70), near lake, "ass. RanunculoEleocharetum acicularis" (Randjelović, V. 27-Aug-1992, Randjelović \& Zlatković 2010: 70), flooded areas, eastern shore, 1200 m, "ass. Elatino triandraeEleocharetum acicularis subass. ranunculetosum aquatilis" + "subass. eleocharetosum acicularis (typicum)” (Randjelović \& Zlatković 2010: 224).

NOTES: The majority of its recent habitats in Serbia is situated primarily in parts of Vojvodina where it is a rather common species in temporary forest ponds and wetland micro-habitats developed on saline pastures (Fig. 7). Protected by law in Serbia (Anonymous, 2010-2016).

Lythrum $\times$ scabrum Simonk., Természetrajzi füz. 1(4): 241 (1877) [salicaria $\times$ virgatum $]$

NEW DATA: Banat: ER 10-EQ 29 Vršac-Margita, near a road, edge of drain ditch filled with water (Šajinović, B. 12-Jul-1972).

PUBLISHED DATA: Banat: Pančevo ["Pancsova"]: DQ 76 Topola: Duboka Topola, wet places [“Topolá”] (Simkovics 1882: 50).

NOTES: By its general habit resembles vigorous L. virgatum, from which differs by middle and lower leaves with slightly truncate or cordate base, epicalyx segments conspicuously longer than sepals and plant colour more dull green (approaching L. salicaria), not at all with glaucous-green hues (which are typical for L. virgatum). Whole plant, especially stem edges, leaf-margins, nerves, calyces and epicalyx segments manifestly scabrid, covered with small, prickly denticles, otherwise perfectly glabrous (our observations, cf. Kmet'ová, 1988: 400). Possibly more widespread in Serbia but confused with L. virgatum (Fig. 8).

Narrow-leaved forms of L. salicaria from saline habitats (e.g. Bački Monoštor-Bezdan: Štrbac, 19-Jul-1999, B. Panjković, PZZP) are approaching this plant, but they are densely and minutely softly hairy throughout (not scabrid-glabrous) with all leaves, including the uppermost, clearly 
cordate or semiamplexicaul at base. This forms are known as L. salicaria [stat. indet.] b. angustifolium Schur, Enum. pl. Transs. 218 (1866).

Lythrum tribracteatum Spreng., Syst. Veg. 4(2): 190 (1827).

NEW DATA: Bačka: Sonta: CR 54 Sveta Ana (Panjković, B. 15-Jul-2004); CR 71-CR 81 Bačka Palanka-Gložan "bumps in the road" (Šajinović, B. 28-Jul1965).

Banat: DR 44 Kumane, saline pastures along the railroad to Melenci, (Perić, R. 16-Sep-2010); Melenci: DR 44 Rusanda, $\approx 45^{\circ} 31^{\prime} 49.90^{\prime \prime} \mathrm{N}, 020^{\circ} 18^{\prime} 03.88^{\prime \prime}$ E, 74 m (Perić, R., Stojšić, V. 13-Jul-2010).

NW Serbia: Special Nature Reserve "Zasavica", field (Perić, R. 02-Aug2004); Bogatić: CQ 77 Ravnje: Staniševac (Perić, R. 16-Jul-2010); CQ 77 Banovo Polje: Ribnjača swamp, 44 $54^{\prime} 47.75^{\prime \prime} \mathrm{N}, 019^{\circ} 24^{\prime} 22.54^{\prime \prime} \mathrm{E}, 77 \mathrm{~m}+$ $44^{\circ} 54$ ' 35. 59” N, 019 24' 27. 38” E, 76 m (Perić, R., Stojšić, V. 22-Jul-2010), [subnom. Lythrum sp.] Lug, field (Perić, R. 01-Aug-2006).

PUBLISHED DATA: Bačka: DR 29 Adorjan [“Adorján”] (Kovács Kümmerle 1917: 53), [subnom. Lythrum bibracteatum Salzm.] vicinity (Kovács 1929: 128); CR 72 Obrovac (Atanacković 1958: 146); DR 20 Čortanovci (Jovanović-Dunjić 1973: 6).

Banat: DR 57 Kikinda ["Nagykikinda"], vicinity ("not very rare" Slavnić

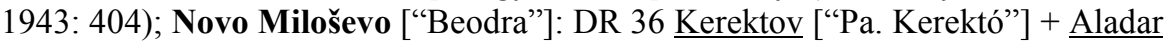
["Pa. Aladár"] (Thaisz 1907: 169).

Srem: Obedska Bara: Kupinovo: DQ 25 Livade Majke Angeline, "ass. Poeto-Alopecuretum pratensis R. Jov. 1957 subass. clematetosum integrifoliae" (Puzović et al. 2008: 22).

Šumadija: Belgrade: DQ 55 Ada Ciganlija, "ass. Populeto-Salicetum" (Rajevski 1950: 174).

NE Serbia: Veliko Gradište: [subnom. Lythrum bibracteatum Salzm.] EQ 35 Kisiljevo: Srebrno jezero ["Kisiljevačka bara"] (Pančić 1874: 302).

IMPRECISE PUBLISHED DATA: Serbia (Gajić 1980a: 128; 1980b: 81); Serbia proper (Jovanović-Dunjić 1973: 6); Vojvodina, "ass. Lythrum tribracteatumLythrum hyssopifolia" (Slavnić 1951: 156); Bačka (Slavnić 1953: 52), "sporadically" (Budak 1998: 92); Banat (Slavnić 1953: 52); Fruška Gora Mt.: on the shores of stagnant waters and in temporary inundated places (Obradović 1966: 49); Koviljski rit (Budak et al. 1992: 49); Novi Sad (Jovanović-Dunjić 1973: 6); “Zasavica" Special Nature Reserve (Perić et al. 2017: 76); Šumadija (Gajić 1967: 184); Kosovo (Jovanović-Dunjić 1973: 6).

NoTES: Rare wetland species occuring in Serbia on flooded abandoned fields, fallows and on saline pastures along cattle watering places (Fig. 8). Its largest and one of the best preserved populations are recorded in "Zasavica" Special Nature Reserve where it is often accompanied with $L$. hyssopifolia L. (ass. Lythrum tribracteatum-Lythrum hyssopifolia Slavnić 1951). Protected by law in Serbia (Anonymous, 2010-2016). 
Phlomis tuberosa L., Sp. Pl. 586 (1753).

NEW DATA: Banat: DQ 89 Padina: Dolina, $45.077572^{\circ} \mathrm{N}, 020.778846^{\circ} \mathrm{E}$, $124 \mathrm{~m}$ (Perić, $R$. 02-Jun-2020) $+45.072705^{\circ} \mathrm{N}, 020.783894^{\circ} \mathrm{E}, 124 \mathrm{~m}+$ $45.070484^{\circ} \mathrm{N}, 020.790861^{\circ} \mathrm{E}, 127 \mathrm{~m}$ (Perić, R., 02-Jun-2020, pers. comm.).

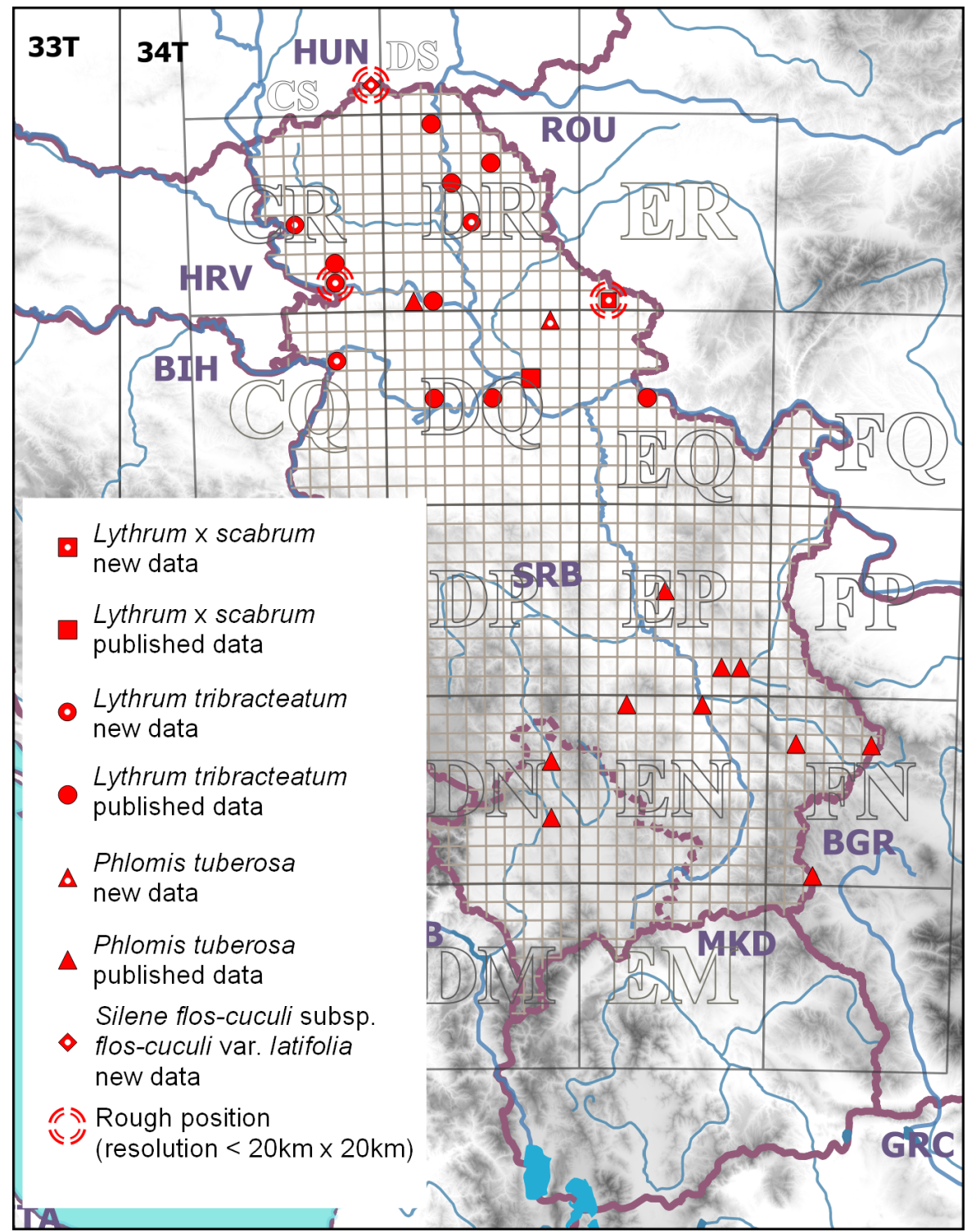

Fig. 8. - New herbarium and published data on the distribution of Lythrum $\times$ scabrum Simonk., L. tribracteatum Spreng., Phlomis tuberosa L. and Silene flos-cuculi [subsp. flos-cuculi] var. latifolia Bolle in Serbia.

PUBLISHED DATA: Srem: Fruška Gora Mt:: DR 10 Partizanski put: flat and sloping terrain along the both sides of Partizanski put road on first few kilometers 
going west from junction with Novi Sad-Belgrade road, $300 \mathrm{~m}$, brownized (degraded) chernozem, on deep loess substrate (Jovanović 1968: 87).

C Serbia: EN 29 Blace (Diklić 1974: 386); Niš: EN 69 Mali Lalinac ["Lalinac", "Lalinačke pojate"]: Lalinački Djeram ["Lalinačka slatina"] (Niketić 1995: 34; 1999: 330; 21-Jun-2000, 26-May-2001, 16-Jun-2002 Zlatković et al.

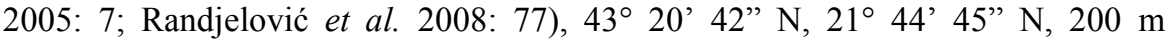
(Zlatković et al. 2014: 95).

Pomoravlje: Paraćin: EP 45 Lešje (Pančić 1856: 513; 1874: 569; Diklić 1974: 386).

E Serbia: EP 71 Pirkovac (Pančić 1856: 513; 1874: 569; Diklić 1974: 386); EP 81 Niševac ["Niševci"] (Pančić 1874: 569; Diklić 1974: 386); Šljivovička Planina Mt.: FN 17 Šljivovik: Stranje: Barkov Del, 800-1000 m, "alliance Festucion valesiacae Klika 31", limestone (Randjelović et al. 2003: 3); Vidlič Mt.: FN 57 Vlkovija, $1100 \mathrm{~m}$ and $1090 \mathrm{~m}$, S exposure (Marković et al. 2015: 65).

SE Serbia: FN 20 Rudina Mt. (Milosavljević \& Randjelović 2002: 33).

Kosovo: Leposavić: [subnom. Phlomoides tuberosa (L.) Moench] DN 86 Slatina ["Ibarska Slatina"], 43 02' 85. 4" N, 020 49' 0. 30" N, 451 m, NE exposure, slope $30^{\circ}$, siliceous ground (Krivošej, Z., Prodanović, D., Stanojević, $M$. 15-Jun-2017 Prodanović et al. 2018: 14); DN 83 Srbica: vicinity (Nikolić et al. 1986: 309); DN 83 Poljance ["Polac"], uncultivated places, meadows edges (Hundozi 1981: 128; 1983-1986: 242).

IMPRECISE PUBLISHED DATA: Serbia (Hayek 1929: 268; Gajić 1980a: 131; 1980b: 80).

NOTES: This interesting steppic plant of striking size has been unknown to Vojvodina for more than 5 decades, in spite of presence of numerous, seemingly adequate habitats in Srem and Banat (Fig. 8). The only one and unconfirmed published record of this species for Vojvodina implies that this is unquestionably a very rare plant, so it was of no little surprise fact that this plant thrives abundantly in the vicinity of Padina with hundreds of individuals (Fig. 9). Contrary to our expectations and beside the most splendid steppic habitats stretching along the whole lenght of almost $7 \mathrm{~km}$ long loess valley Dolina near Padina, we found this species concentrated at only 3 localities characterized with moderately disturbed vegetation which could be provisionaly placed within alliance Festucion rupicolae Soó. Accompanying species were: Achillea pannonica Scheele, Capsella bursapastoris (L.) Medik., Carduus nutans L., Chrysopogon gryllus (L.) Trin., Convolvulus arvensis L., Cruciata pedemontana (Bellardi) Ehrend., Dactylis glomerata L., Euphorbia nicaeensis subsp. glareosa (Pallas ex M. Bieb.) Radcl.-Sm., Festuca rupicola Heuff., Filipendula vulgaris Moench, Fragaria viridis Weston, Galium glaucum L., Koeleria macrantha (Ledeb.) Schult., Medicago minima (L.) L., Muscari comosum (L.) Mill., Myosotis arvensis (L.) Hill., Plantago lanceolata L., Potentilla argentea L., Stachys recta L., Teucrium chamaedrys L., Thymus pulegioides subsp. pannonicus 
(All.) Kerguélen, Tragopogon dubius Scop., Valerianella dentata (L.) Pollich and Verbascum phoeniceum L. These 3 localities are situated in the upper, more distant and regularly mowed part of loess valley without or almost without grazing (in contrast with lower, heavily grazed part of valley adjacent to the village where this species does not occur). At each of aforesaid localities the number of non-flowering rosettes is about 3 to 4 times surpassing the number of flowering individuals: I locality $\left(45.077572^{\circ} \mathrm{N}, 020.778846^{\circ} \mathrm{E}\right)-136$ flowering individuals, II $\left(45.072705^{\circ}\right.$ $\left.\mathrm{N}, 020.783894^{\circ} \mathrm{E}\right)-$ more than 100 flowering individuals, III $\left(45.070484^{\circ}\right.$ $\mathrm{N}, 020.790861^{\circ} \mathrm{E}$ )-more than 100 flowering individuals. This species is protected by law in Serbia (Anonymous, 2010-2016).

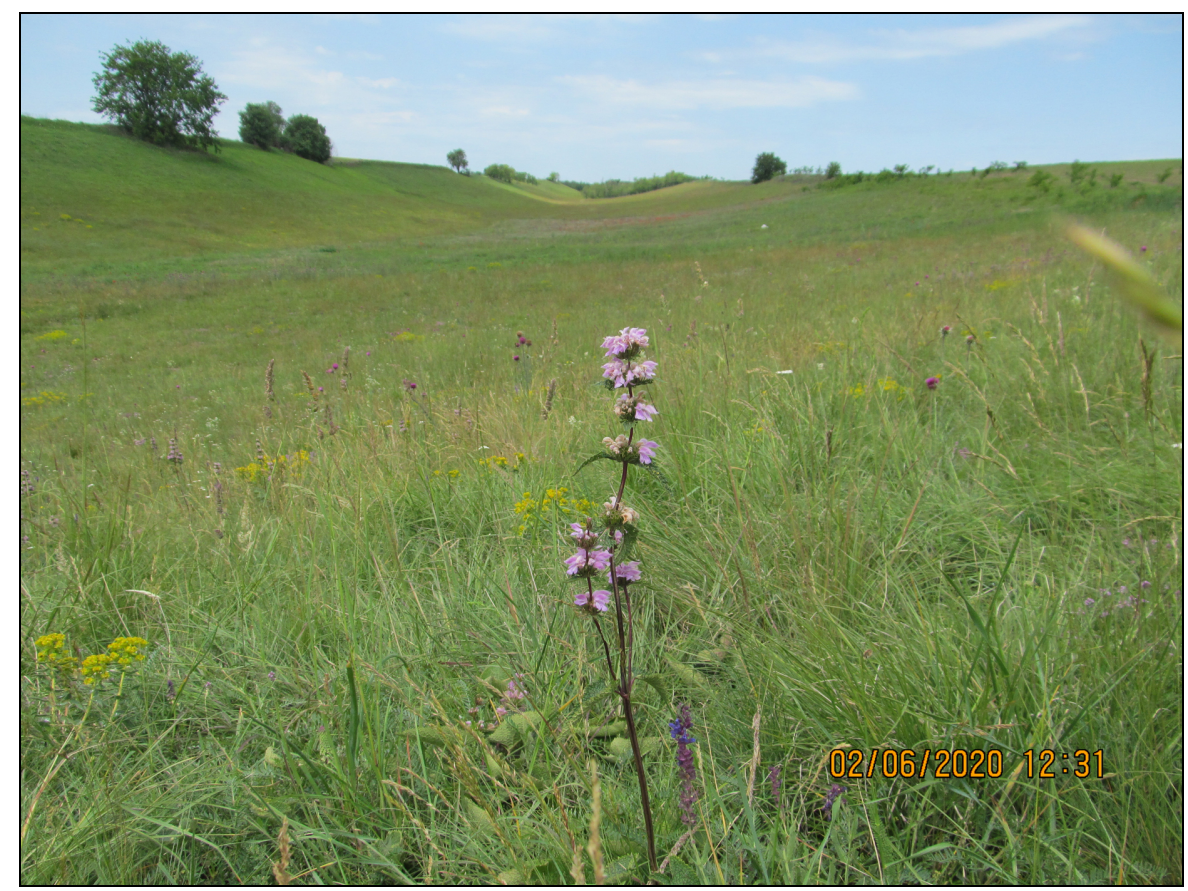

Fig. 9. - Phlomis tuberosa L., vicinity of Padina, 02. 06. 2020. (photo R. Perić).

Silene flos-cuculi (L.) Clairv., Man. Herbor. Suisse 146 (1811) [subsp. flos-cuculi] var. latifolia Bolle [sub Lychnide!], Verh. bot. Verein. Brandenb. 7: 19 (1865).

NEW DATA: Bačka: CS 91-DS 01 Subotica-Horgoš Sands ["Subotičke šume"] (Butorac, B. 18/22-May-1998).

NOTES: New for Serbia (Fig. 8). More robust plant with oblong-lanceolate to obovate leaves $10-16 \mathrm{~mm}$ wide (vs. up to $10 \mathrm{~mm}$ wide in var. floscuculi). Plant usually matt green without pervading reddish stem coloration (Bolle 1865: 19; Soó 1970a: 302). Possibly more widespread in Serbia. 


\section{CONCLUSIONS}

Article presents a third part of data selected from the most recent results of our revision of vascular plant material deposited at the Herbarium collection of the Institute for Nature Conservation of the Vojvodina province (PZZP). Selected plant taxa include 7 genera (Filago L., Ludwigia L., Luzula DC., Lycopus L., Lythrum L., Phlomis L. and Silene L.), 11 species, three subspecies, three varieties, one infraspecific taxon with undetermined status [stat. indet.] and one nothospecies within 26 UTM squares.

New taxa for Serbia are: two varieties (Luzula campestris [subsp. campestris] var. elata, Silene flos-cuculi [subsp. flos-cuculi] var. latifolia) and one "stat. indet." taxon (Luzula multiflora [subsp. multiflora] [stat. indet.] b. uliginosa). Confirmed taxa for Serbia are: species (Ludwigia palustris) and nothospecies (Lythrum $\times_{\text {scabrum }}$ ) and for Vojvodina: 1 species (Phlomis tuberosa).

New and precise chorological data are given for seven species (Filago minima, Ludwigia palustris, Luzula exspectata, L. taurica, Lythrum portula, L. tribracteatum, Phlomis tuberosa), 1 variety (Lycopus europaeus var. mollis) and 1 nothospecies (Lythrum $\times$ scabrum).

Nationally protected taxa include three species (Lythrum portula, $L$. tribracteatum, Phlomis tuberosa) categorized as "protected".

\section{REFERENCES}

Adamović L. (1898): Die Vegetationsformationen Ostserbiens. - Botanische Jahrbücher für Systematik, Pflanzengeschichte und Pflanzengeographie 26(1): 124-218. [in German]

Adamović L. (1899): Die mediterranen Elemente der serbischen Flora. - Botanische Jahrbücher für Systematik, Pflanzengeschichte und Pflanzengeographie 27(12): 351-389. [in German]

Adamović, L. (1908): Flora jugoistočne Srbije/ Flora Serbiae austro-orientalis. Rad Jugoslavenske akademije znanosti i umjetnosti knjiga 175, matematičkoprirodoslovni razred. 44: 153-214. [in Serbo-Croatian]

Adamović, Ž. R. (1959): The morrocan locust (Dociostaurus maroccanus Thunberg) in North Banat, Serbia. - Glasnik Prirodnjačkog muzeja u Beogradu, serija B 13: 5-123.

Amidžić, L., Krivošej, Z. (1996): August Aspect of the High-mountain Vascular Flora of the Djeravica Volcanic Massif. - The University thought, publication in natural sciences, University of Pristina, Serbia 3(2): 27-32.

Amidžić, L., Stevanović, V. (1996): Saxifragetum cymosae- a New Chasm-phyte Community on the Metohian Prokletije. - The University thought, publication in natural sciences, University of Pristina, Serbia 3(2): 33-39. 
Amidžić, L. (1997): Pinetum mughi-Geum bulgaricum, New Association of the Mountain Pine (Pinus mugoTurra) on Prokletije Mountain. - The University thought, publication in natural sciences, University of Pristina, Serbia 4(2): 89-95.

Amidžić, L., Krivošej, Z. (1998): High-mountain Flora of Nedzinat and Zuti Kamen (Prokletije Mt.). - The University thought, publication in natural sciences, University of Pristina, Serbia 5(1): 99-112.

Amidžić, L., Krivošej, Z., Ranđelović, V. (1999): O kalcifilnoj flori severozapadnog dela Šar-planine. - Zaštita prirode 51(2): 61-67. [in Serbian]

Amidžić, L., Ostojić, D. (2006): Prilog poznavanju vegetacije Šar-planine. - Zaštita prirode 56(2): 33-49. [in Serbian]

Andrés-Sánchez, S., Martínez-Ortega, M. M., Rico, E. (2013): Taxonomic revision of the genus Logfia (Asteraceae, Gnaphalieae) in the Mediterranean region. Anales del Jardín Botánico de Madrid 70(1): 7-18.

Anonymous (2010-2016): Rulebook on declaration and protection of protected and strictly protected species of plants, animals and fungi. - Official Gazette of Republic of Serbia 5/10, 88/10, 91/10, 47/11, 14/16, 98/16. [in Serbian]

Atanacković, N. (1958): Prilog flori Bačke. - Matica srpska, zbornik za prirodne nauke 14: 143-149. [in Serbian]

Bačič, T., Frajman, B., Dolenc Koce, J. (2015): Diversification of Luzula sect. Luzula (Juncaceae) on the Balkan Peninsula-a cytogenetic approach. In: Bogdanović, S., Jogan, N. (ed.): Book of abstracts, $6^{\text {th }}$ Balkan Botanical Congress, Rijeka, September 14-18, 2015: 47-48. - Natural History Museum Rijeka, Croatian Botanical Society, Botanical Society of Slovenia, University of Rijeka.

Bačič, T., Frajman, B., Dolenc Koce, J. (2016): Diversification of Luzula sect. Luzula (Juncaceae) on the Balkan Peninsula - a cytogenetic approach. - Folia Geobotanica 51(1): 51-63.

Bačič, T., Dolenc Koce, J., Frajman, B. (2019): Diversification and distribution patterns of Luzula sect. Luzula (Juncaceae) in the Eastern Alps: a cytogenetic approach combined with extensive herbarium revisions. - Alpine Botany 129: 149-161.

Berisha, N., Ćušterevska, R., Millaku, F., Kostadinovski, M., Matevski, V. (2020): Contribution to the knowledge on the flora of Mt Luboten, Sharri Mts., Kosovo. - Thaiszia-Journal of Botany (Košice) 30(2): 115-160.

Biró, M., Molnár, Zs., Babai, D., Dénes, A., Fehér, A., Barta, S., Sáfián, L., Szabados, K., Kiš, A., Demeter, L., Öllerer, K. (2019): Reviewing historical traditional knowledge for innovative conservation management: A reevaluation of wetland grazing. - Science of The Total Environment 666: 1114-1125.

Biró, M., Molnár, Zs., Öllerer, K., Lengyel, A., Ulicsni, V., Szabados, K., Kiš, A., Perić, R., Demeter, L., Babai, D. (2020): Conservation and herding co-benefit from traditional extensive wetland grazing. - Agriculture, Ecosystems and Environment 300. 15 September 2020, 106983

Bolle, C. (1865): Kleine Nachträge zu Ascherson's Flora. - Verhandlungen des botanischen Vereins für die Provinz Brandenburg und die angrenzenden Länder 7: 15-29. [in German] 
Broz, V. (1951): Flora deliblatske peščare. - Zaštita prirode 2-3: 318-342. [in Serbian]

Budak, V., Boža, P., Igić, R. (1992): Neke retke, reliktne i ugrožene biljke Koviljskog rita. - Univerzitet u Novom Sadu, zbornik radova Prirodno-matematičkog fakulteta, serija za biologiju 22: 49-53. [in Serbian]

Budak, V. (1998): Flora i biljnogeografske odlike flore slatina Bačke. - Matica srpska, odeljenje za prirodne nauke, Novi Sad. [in Serbian]

Čanak, M., Gajić, M., Korać, M. (1979): Srednjeevropski i subsrednjeevropski florni elemenat u flori Srbije. - Glasnik Prirodnjačkog muzeja u Beogradu, serija B 34: 21-29. [in Serbian]

Černjavski, P. (1950): Morfološko-ekološka analiza roda Thymus iz okoline Beograda. - Glasnik Prirodnjačkog muzeja srpske zemlje, serija B 3-4: 113-165. [in Serbian]

Demeter, L., Kiš, A., Biró, M., Babai, D., Szabados, K., Marinkov, J., Juhász, E., Ulicsni, V., Kemenes, A., Molnár, Zs. (2018): Traditional pig herding in the floodplain of the Sava river-an ecological perspective of forest and wetland grazing in Bosut forest, Serbia. In: Anačkov, G., Zorić, L. (ed.): Book of Abstracts, $7^{\text {th }}$ Balkan Botanical Congress, Book of Abstracts, September, 1014. 2018., Novi Sad, Serbia. - Department of Biology and Ecology, Novi Sad, Botanical Society “Andreas Wolny”, Novi Sad. - Botanica Serbica (Supplement 1) $42: 118$.

Diklić, N., Nikolić, V. (1961): Novi podaci o nalazištu biljnih vrsta u Srbiji. Glasnik Prirodnjačkog muzeja u Beogradu, serija B 17: 215-234. [in Serbian]

Diklić, N. (1974): Rod Phlomis L. In: Josifović, M. (ed.): Flora SR Srbije 6: $383-$ 386. - Srpska akademija nauka i umetnosti, Beograd. [in Serbian]

Dítě, D., Eliáš Jr., P., Dítě, Z., Šimková, A. (2017): Recent distribution and phytosociological affiliation of Ludwigia palustris in Slovakia. - Acta Societatis Botanicorum Poloniae 86(1): 35-44.

Domac, R. (1950): Flora za određivanje i upoznavanje bilja. - Izdavački zavod Jugoslavenske akademije znanosti i umjetnosti, Zagreb. [in Croatian]

Duraki, Š., Stanojević, M., Stojanović, V. (2017): Florističke karakteristike šarplaninskog grebena Kobilica. - Zaštita prirode 67(1-2): 5-23. [in Serbian]

Euro+Med (2006+): Euro+Med PlantBase-the information resource for EuroMediterranean plant diversity. [http://ww2.bgbm.org/EuroPlusMed/] (accessed: September $\left.15^{\text {th }} 2019\right)$.

Flora Croatica Database $(2004+)$ : [https://hirc.botanic.hr/fcd/] (accessed: November $\left.10^{\text {th }} 2020\right)$.

Formánek, E. (1895): Zweiter Beitrag zur Flora von Serbien und Macedonien. Verhandlungen des naturforschenden Vereines in Brünn 34: 255-365. [in German]

Fritsch, K. (1918): Neue Beiträge zur Flora der Balkanhalbinsel, inbesondere Serbiens, Bosniens und Herzegowina. - Mitteilungen des Naturwissenschaftlichen Vereines für Steiermark 54: 235-299. [in German]

Gajić, R. M. (1965): Prilog poznavanju flore srednje i južne Šumadije. - Glasnik muzeja šumarstva i lova 5: 7-54. [in Serbian] 
Gajić, M. R. (1967): Florni elementi Šumadije. - Zaštita prirode 34: 171-201. [in Serbian]

Gajić, R. M. (1973): Prilog poznavanju flore Jugoslavije. - Glasnik Prirodnjačkog muzeja u Beogradu, serija B 28: 5-33. [in Serbian]

Gajić, M. (1975): Fam. Asteraceae Dumortier. In: Josifović, M. (ed.): Flora SR Srbije 7: 1-475. - Srpska akademija nauka i umetnosti, Beograd. [in Serbian]

Gajić, M. (1980a): Pregled vrsta flore SR Srbije sa biljnogeografskim oznakama. Glasnik Šumarskog fakulteta, Beograd. 54: 111-141. [in Serbian]

Gajić, M. (1980b): Pontsko-centralnoazijski florni elementi u flori SR Srbije. Glasnik Šumarskog fakulteta, Beograd. 54: 79-90. [in Serbian]

Gajić, M. (1989): Flora i vegetacija Golije i Javora. - Šumarski fakultet-Beograd, OOUR Šumarstvo „Golija“, Ivanjica. [in Serbian]

Gajić, M., Obratov, D., Purić, D. (1991): Prilog poznavanju flore Kopaonika. Glasnik Šumarskog fakulteta (Beograd) 73: 647-656. [in Serbian]

Grdinić, B., Boža, P., Knežević, A., Krivokućin, I. (2001): Pregled flore zapadne Bačke. - Norma (Sombor) 7(1-2): 137-157. [in Serbian]

Grebenščikov, O. (1943): Prilog poznavanju vegetacije planine Koprivnik kod Peći. In: Ohridski zbornik 11: 241-267. - Srpska Kraljevska Akademija. Posebna izdanja CXXXVI. Prirodnjački i matematički spisi. 35. [in Serbian]

Gremli, A. (1878): Excursionflora für die Schweiz. - Druck und Verlag von J. J. Christen, Aarau. [in German]

Grisebach, A. (1844): Spicilegium Florae rumelicae et bithynicae exhibens synopsin plantarum quas aest. 1839 legit auctor A. Grisebach. 2. - Prostat apud Fridericum Vieweg et filium, Brunsvigae.

Guelmino, J. (1968): Zenta és környékének növényei. I. Virágosok. - Građa za Monografiju Sente br. 12, Novi Sad-Senta. [in Hungarian]

Hayek, A. (1926): Prodromus Florae peninsulae Balcanicae 1(5-6). - Feddes Repert. (Beih.) 30(1): 673-960.

Hayek, A. (1929): Prodromus Florae peninsulae Balcanicae 2(3). - Feddes Repert. (Beih.) 30(2): 241-336.

Hayek, A. (1931): Prodromus Florae peninsulae Balcanicae 2(5). - Feddes Repert. (Beih.) 30(2): 577-768.

Herbarium HAL, the Martin Luther University of Halle-Wittenberg [https:// herbarium.univie.ac.at/index.htm] (accessed: September $05^{\text {th }} 2020$ ).

Herbarium WU, Institute of Botany, University of Vienna - Institute of Botany, University of Vienna. [http://herbarium.univie.ac.at] (accessed: September $05^{\text {th }}$ 2020).

Hundozi, B. (1981): Përsëri mbi disa lloje bimore të florës së Kosovës. Univerzitet Kosova, Prirodno-matematički fakultet, zbornik radova 7: 127-131. [in Albanian]

Hundozi, B. (1983-1986): Flora e livadheve ultësinore të Kosovës. - Univerzitet Kosova, Prirodno-matematički fakultet, zbornik radova 9: 235-251. [in Albanian]

IUCN (2012): IUCN Red List Categories and Criteria: Version 3.1. Second edition. - IUCN, Gland, Switzerland \& Cambridge, UK. 
Ivančević, B., Savić, S., Sabovljević, M., Niketić, M., Tomović, G., Zlatković, B., Ranđelović, V., Lakušić, D., Ćetković, A., Pavićević, D., Krpo-Ćetković, J., Crnobrnja-Isailović, J., Puzović, S., Paunović, M. (2007): Pregled vrsta Stare planine u Srbiji. In: Lakušić, D., Ćetković, A. (ed.): Biodiverzitet Stare planine u Srbiji-Rezultati projekta: „Prekogranična saradnja kroz upravljanje zajedničkim prirodnim resursina-Promocija umrežavanja i saradnje između zemalja Jugoistočne Evrope: 157-221. - Regionalni centar za životnu sredinu za Centralnu i Istočnu Evropu, Kancelarija u Srbiji, Beograd. [in Serbian]

Janković, M. M. (1972): Ekološka studija problema zarašćivanja veštačkih jezera na primeru budućeg jezera na Novom Beogradu. - Glasnik Instituta za botaniku i botaničke bašte Univerziteta u Beogradu 7(1-4): 153-195. [in Serbian]

Janković, M. M. (1985): Makrofite naše zemlje i mogućnosti proizvodnje i eksploatacije njihove biomase. - Glasnik Instituta za botaniku i botaničke bašte Univerziteta u Beogradu 19: 107-168. [in Serbian]

Jotić, B., Marković, M., Petrović, B., Fusijanović, I., Pavlović, D., Ranđelović, V. (2011): The vascular flora of the Vučje hill near Pirot city (Eastern Serbia). Biologica Nyssana 2(2): 91-106.

Jotić, B., Miljković, M., Marković, M., Zlatković, B., Ranđelović, V. (2013): The vascular flora of the Tepoš plateau around Pirot city (Eastern Serbia). Biologica Nyssana 4(1-2): 19-33.

Jovanović, B. (1968): Neke eko-i cenološke karakteristike domaće stepske višnjeCerasus fruticosa (Pall.) Woronow. - Glasnik Šmarskog fakulteta (Beograd) 34: 83-90. [in Serbian]

Jovanović-Dunjić, R. (1971): Ispitivanja strukture i ekoloških uslova fitocenoza u jednom mozaikkompleksu na tresavama Stare planine. - Glasnik Instituta za botaniku i botaničke bašte Univerziteta u Beogradu 6(1-4): 91-106. [in Serbian]

Jovanović-Dunjić, R. (1973): Fam. Lythraceae Lindl. In: Josifović, M. (ed.): Flora SR Srbije 3: 1-8. - Srpska akademija nauka i umetnosti, Beograd. [in Serbian]

Katić, D. (1910): Vlasinska tresava i njezina prošlost. - Spomenik Srpske Kraljevske Akademije, prvi razred 50(8): 14-56. [in Serbian]

Kerner, A. (1866): Descriptiones plantarum novarum. - Österreichische botanische Zeitschrift 16: 370-371.

Kirschner, J. (1991): An account of natural hybridization within Luzula sect. Luzula (Juncaceae) in Europe. - Preslia (Praha) 69: 81-112.

Kirschner, J. (2002): Juncaceae 1: Rostkovia to Luzula. Species plantarum. Flora of the world. 6. - Australian Biological Resources Study, Canberra.

Kiš, A., Szabados, K., Demeter, L., Biró, M., Perić, R., Marinkov, J., Molnár, Z. (2019): Traditional pig-herding as suitable habitat management of endangered wetland species. In: Ranđelović, V., Stojanović-Radić, Z., Nikolić, D. (ed.): Book of abstracts, $13^{\text {th }}$ Symposium on the Flora of Southeastern Serbia and Neighboring Regions, Stara Planina Mt., $13^{\text {th }}$ to $23^{\text {th }}$ June, 2019: 81-82. Department of Biology and Ecology, Faculty of Science and Mathematics, University of Niš \& Institute for Nature Conservation of Serbia, Belgrade. 
Kmet’ová, E. (1988): Lythraceae Lindl. In: Bertová, L. (ed.): Flóra Slovenska. 4(4): 389-403. - VEDA, vydvatel'stvo Slovenskej akadémie vied, Bratislava. [in Slovakian]

Knežević, A. (1994): Monografija flore vaskularnih biljaka na slatinama u regionu Banata (Jugoslavija). - Matica srpska, Novi Sad. [in Serbian]

Košanin, N. (1908): Daićsko jezero na Goliji. Hidrobiološka studija. - Glas Srpske kraljevske akademije 75: 1-50. [in Serbian]

Košanin, N. (1910a): Elementi Vlasinske flore (Algae, Bryophyta, Pteridophyta et Phanerogamae). - Muzej srpske zemlje (Beograd), posebno izdanje 10: 1-42. [in Serbian]

Košanin, N. (1910b): Vlasina biljnogeografska studija. - Glas Srpske Kraljevske Akademije 81: 86-186. [in Serbian]

Kovács, F. (1915): Változások Óbecse flórájában. - Botanikai közlemények 14(12): 68-76. [in Hungarian]

Kovács, F. (1929): Óbecse határának viragos növényei. - Szeged városi nyomda és könyvkiadó, R.T. Szeged. [in Hungarian]

Kümmerle, J. B. (ed.) (1917): Növénytani repertorium: Gyüjtemények. In: . Botanikai közlemények 16(1-3): 51-53. [in Hungarian]

Lakušić, R. (1968): Planinska vegetacija jugoistočnih Dinarida. - Glasnik Republičkog zavoda za zaštitu prirode i prirodnjačke zbirke u Titogradu 1: 9-75. [in Serbian]

Lakušić, D. (1996): Pregled flore Kopaonika (JZ Srbija, Jugoslavija). - Acta biologica iugoslavica, serija D, Ekologija 31(2): 1-35. [in Serbian]

Lampinen, R. (2001): Universal Transverse Mercator (UTM) and Military Grid Reference System (MGRS). [http://www.luomus.fi/english/botany/afe/map/ utm.htm] (accessed: September 15 $5^{\text {th }}$ 2019).

Lazarević, P. (2014): Florističke odlike područja Peštersko polje na Pešterskoj visoravni (jugozapadna Srbija). - Zaštita prirode 64(1): 11-20. [in Serbian]

Marković, M., Nikolić, B., Rakonjac, Lj., Pešić, D. (2015): Vegetacijske odlike suvih pašnjaka i kamenjara planine Vidlič (jugoistočna Srbija). - Glasnik Šumarskog fakulteta (Beograd) 112: 59-82. [in Serbian]

Marković, M., Rakonjac, Lj., Smiljić, M., Papović, O. (2018): Termofilne hrastove šume i šibljak grabića treće godine nakon požara na planini Vidlič. - Šumarstvo (Beograd) 70(1-2): 99-110. [in Serbian]

Milosavljević, V., Ranđelović, N. (2002): Prilog poznavanju florističkog i vegetacijskog diverziteta Rudina planine kraj Bosilegrada. In: Anonymous (ed.): Zbornik rezimea, 7. Simpozijum o flori jugoistočne Srbije i susednih područja sa međunarodnim učešćem, Dimitrovgrad, 2002: 47. - Prirodno-matematički fakultet u Nišu, Institut za botaniku Bugarske akademije nauka, Biološko društvo „Dr Sava Petrović“, Bugarsko botaničko društvo, Tehnološki fakultet u Leskovcu, DD „Zdravlje“"Leskovac. [in Serbian]

Mišić, V., Jovanović-Dunjić, R., Popović, M., Borisavljević, Lj., Antić, M., Dinić, A., Danon, J., Blaženčić, Ž. (1978): Biljne zajednice i staništa Stare planine. Srpska akademija nauka i umetnosti, posebna izdanja, knjiga DXI, odeljenje prirodno-matematičkih nauka, knjiga 49, Beograd. [in Serbian] 
Moesz, G. (1916): Botanizálás a Száva partján 1915. év nyarán. - Botanikai közlemények 15(1-2): 1-9. [in Hungarian]

Ničić, Đ. I. (1893): Građa za floru okoline Vranje. - Nastavnik (Beograd) 4(1-4): 178. [in Serbian]

Niketić, M. (1995): Pregled flore šireg područja Lalinačke slatine kod Niša. In: Anonymous (ed.): Zbornik rezimea, II Simpozijum o flori Srbije (IV Simpozijum o flori jugoistočne Srbije) 1995: 34. - Vranje. [in Serbian]

Niketić, M. (1999): Aster oleifolius (Lam.) Wagenitz. In: Stevanović, V. (ed.): The Red Data Book of Flora of Serbia 1, Extinct and critically endangered taxa: 329-331. - Ministry of Environment of the Republic of Serbia, Faculty of Biology, University of Belgrade, Institution for Protection of Nature of the Republic of Serbia, Belgrade. [in Serbian]

Niketić, M., Tomović, G., Perić, R., Zlatković, B., Anačkov, G., Djordjević, V., Jogan, N., Radak, B., Duraki, Š., Stanković, M., Kuzmanović, N., Lakušić, D., Stevanović, V. (2018a): Material on the annotated checklist of vascular flora of Serbia. Nomenclatural, taxonomic and floristic notes I. - Bulletin of the Natural History Museum 11: 101-180.

Niketić, M., Tomović, G., Anačkov, G., Djordjević, V., Lakušić, D., Randjelović, V. (2018b): Katalog taksona. In: Niketić, M., Tomović, G. (ed.): Kritička lista vrsta vaskularne flore Srbije 1, Lycopodiopsida, Polypodiopsida, Gnetopsida, Pinopsida i Liliopsida: 57-140. - Srpska akademija nauka i umetnosti, Posebna izdanja, knjiga DCXC, Odeljenje hemijskih i bioloških nauka, knjiga 10, Beograd. [in Serbian]

Nikolić, V. (1976): Fam. Juncaceae A. L. Juss. In: Josifović, M. (ed.): Flora SR Srbije 8: 116-151. - Srpska akademija nauka i umetnosti, Beograd. [in Serbian]

Nikolić, V., Sigunov, A., Diklić, N. (1986): Dopuna flori SR Srbije novim podacima o rasprostranjenju biljnih vrsta. In: Sarić, M. R. (ed.): Flora SR Srbije, Dodatak (2) 10: 257-336. - Srpska akademija nauka i umetnosti, Beograd. [in Serbian]

Obradović, M. (1966): Biljnogeografska analiza flore Fruške gore. - Matica srpska, odeljenje za prirodne nauke, Novi Sad. [in Serbian]

Obradović, M., Boža, P. (1986): Prodromus flore papratnica i semenica Subotičke peščare i bliže okoline. - Univerzitet u Novom Sadu, zbornik radova Prirodnomatematičkog fakulteta, serija za biologiju 16: 121-142. [in Serbian]

Pančić, J. (1856): Verzeichniss der in Serbien wildwachsenden Phanerogamen, nebst den Diagnosen einiger neuer Arten. - Verhandlungen des ZoologischBotanischen Vereins in Wien 6: 475-598. [in German]

Pančić, J. (1874): Flora kneževine Srbije. - Državna štamparija, Beograd. [in Serbian]

Papović, O., Miljković, M., Ranđelović, N., Ranđelović, V. (2014): Analysis of the flora of Rogozna Mountain in Southwestern Serbia: 17-30. [in Serbian]

Perić, R., Stojšić, V., Rilak, S., Škondrić, S. (2016): The account of Elatine ambigua Wight., E. triandra Schkuhr and E. hungarica Moesz collected in Vojvodina (Serbia). - Bulletin of the Natural History Museum (Belgrade) 9: 81-93. 
Perić, R., Stanković, M., Stojšić, V. (2017): Najznačajniji predstavnici vaskularne flore u Specijalnom rezervatu prirode „Zasavica“. In: Simić, S. (ed.): “Zasavica 2017“, Naučno-stručni skup o biodiverzitetu i drugim vrednostima rezervata Zasavica, 23-24. novembar 2017: 73-83. - Pokret gorana Sremska Mitrovica. [in Serbian]

Perić, R., Knežević, J., Škondrić, S. (2018): Materials for a flora of Serbia from the Herbarium collection PZZP (1). - Bulletin of the Natural History Museum (Belgrade) 11: 63-99.

Perić, R., Knežević, J. (2019): Materials for a flora of Serbia from the Herbarium collection PZZP (2). - Bulletin of the Natural History Museum (Belgrade) 12: 85-151.

Prodán, Gy. (1911): Alyssum linifolium Steph. Magyarországon és néhány adat Bácska florájához. - Magyar Botanikai Lapok 10(8-10): 325-329. [in Hungarian]

Prodán, Gy. (1914): Bács-Bodrog-vármegye sziki növényei/ Die Halophytenflora des Komitates Bács-Bodrog. - Magyar Botanikai Lapok 13(1-5): 96-138. [in Hungarian/German]

Prodán, Gy. (1915): Bács-Bodrog vármegye flórája. - Magyar Botanikai Lapok 14(5-12): 120-269. [in Hungarian]

Prodanović, D., Krivošej, Z., Stanojević, M., Ćirić, S. (2018): Supplement to the phytogeographical studies of the rare and internationally significant species in the flora of Serbia, Kosovo and Metohija North. - The University thought, publication in natural sciences, University of Pristina, Serbia 8(1): 10-16.

Puzović, S., Stojšić, V., Dobretić, V. (2008): Monitoring živog sveta vlažnih staništa SRP „Obedska bara“ u procesu revitalizacije. In: Belij, S., Panjković, B. (ed.): Zbornik izvoda, I simpozijum Zaštita prirode u Srbiji, 1-3. oktobar 2008: 22-23. - Zavod za zaštitu prirode, Novi Sad. [in Serbian]

Rajevski, L. (1950): Vegetacija na Adi Ciganliji. - Glasnik Prirodnjačkog muzeja srpske zemlje, serija B 3-4: 167-174. [in Serbian]

Rajevski, L. (1990): Fitocenološke karakteristike planinskih pašnjaka severnog dela Šarplanine. - Glasnik Instituta za botaniku i botaničke bašte Univerziteta $u$ Beogradu 9: 1-62. [in Serbian]

Ranđelović, N., Rexhepi, F., Jovanović, V. (1979): Biljne zajednice severoistočnog Kosova. In: Rauš, Đ. (ed.): Drugi kongres ekologa Jugoslavije, Zadar-Plitvice, 1-7. X. 1979: 957-995. - Savez društava ekologa Jugoslavije-Šumarski fakultet, Zagreb. [in Serbian]

Ranđelović, N., Stamenković, V. (1991): Dve biljne zajednice brdskih pašnjaka na granitima Rujan planine u južnoj Srbiji. - Glasnik Prirodnjačkog muzeja u Beogradu, serija B 46: 23-37. [in Serbian]

Ranđelović, V., Zlatković, B., Amidžić, L. (1998): Flora i vegetacija visokoplaninskih tresava Šar planine. - Zaštita prirode 50: 377-387. [in Serbian]

Ranđelović, V., Zlatković, B., Jušković, M. (2003): Astragalus wilmottianus Stoj. nova vrsta u flori Srbije. In: Ranđelović, V. (ed.): Zbornik radova, 7. Simpozijum o flori jugoistočne Srbije i susednih područja sa međunarodnim učešćem, Dimitrovgrad, 05-09. jun 2002: 1-4. - Odsek za biologiju sa ekologijom Prirodno-matematičkog fakulteta Univerziteta u Nišu, Institut za 
botaniku Bugarske akademije nauka, Biološko društvo „Dr Sava Petrović““, Bugarsko botaničko društvo, Niš. [in Serbian]

Ranđelović, V., Zlatković, B., Dimitrijević, D. (2008): Fitogeografska analiza flore Lalinačke slatine. In: Ranđelović, V. (ed.): Zbornik radova, 9. Simpozijum o flori jugoistočne Srbije i susednih područja sa međunarodnim učešćem, Niš, 01.-03. septembar 2007: 73-82. - Odsek za biologiju sa ekologijom Prirodnomatematičkog fakulteta Univerziteta u Nišu, Biološko društvo "Dr Sava Petrović”, Niš. [in Serbian]

Ranđelović, V. N., Zlatković, B. K. (2010): Flora i vegetacija Vlasinske visoravni. - Odsek za biologiju i ekologiju, Prirodno-matematički fakultet, Univerzitet u Nišu, Niš. [in Serbian]

Rechinger, K. H. fil. (1935): Ergebnisse einer botanischen Reise in den Bertiscus (Nordalbanische Alpen). - Feddes Repertorium specierum novarum regni vegetabilis 38(13-25): 138-152, 319-389. [in German]

Rechinger, K. H. (1952): Phytographische Notizen. - Godišnjak Biološkog Instituta u Sarajevu 5(1-2): 335-338. [in German]

Rochel, A. (1838): Botanische Reise in das Banat im Jahre 1835. - Gustav Heckenast, Pesth.

Schlechtendal, D. F. L. de (1823): Flora Berolinensis. Pars prima: Phanerogamia. 1. - Sumtibus Ferdinandi Dümmler, Berolini.

Schlosser, J. C., Vukotinović, L. (1869): Flora croatica. - Apud Fr. Župan (Albrecht et Fiedler), Zagrabiae.

Schulzer von Mueggenburg, S., Kanitz, A., Knapp, J. A. (1866): Die bisher bekannten Pflanzen Slavoniens. - Carl Czermak, Wien. [in German]

Simkovics, L. (1882): Pancsova vidékének növényzete. - Magyar Növénytani Lapok 6 [64-65]: 49-53. [in Hungarian]

Slavnić, Ž. (1943): Adatok az alsó Tiszavidék flórájának ismerethéz. - Botanikai Közlemények 40: 400-405. [in Hungarian]

Slavnić, Ž. (1950-1952): Prilog flori našeg Podunavlja. - Glasnik Biološke sekcije (Periodicum biologorum) (Hrvatsko prirodoslovno društvo), serija II/B 4-6: 145177. [in Serbian]

Slavnić, Ž. (1951): Pregled nitrofilne vegetacije Vojvodine. - Naučni zbornik Matice srpske. 1: 84-169. [in Serbian]

Slavnić, Ž. (1953): Biljnogeografska analiza i florogeneza sremske halofitske vegetacije. - Zbornik Matice srpske, serija prirodnih nauka 4: 35-64. [in Serbian]

Soó, R. (1968): A magyar flóra és vegetáció rendszertani-növényföldrajzi kézikönyve. 3. - Akadémiai kiadó, Budapest. [in Hungarian]

Soó, R. (1970a): A magyar flóra és vegetáció rendszertani-növényföldrajzi kézikönyve. 4. - Akadémiai kiadó, Budapest. [in Hungarian]

Soó, R. (1970b): Species et combinationes novae florae Europae praecipue Hungariae IX. - Acta botanica Hungarica 16: 363-372. [in Hungarian]

Soó, R. (1973): A magyar flóra és vegetáció rendszertani-növényföldrajzi kézikönyve. 5. - Akadémiai kiadó, Budapest. [in Hungarian] 
Stevanović, V., Jovanović, S., Janković, M. M. (1995): Prilog rasprostranjenju i ekologiji visokoplaninskih borova na Šarplanini. - Glasnik Instituta za botaniku i botaničke bašte Univerziteta u Beogradu 28: 91-99. [in Serbian]

Stevanović, V. (ed.) (1999): The Red Data Book of Flora of Serbia 1, Extinct and critically endangered taxa. - Ministry of Environment of the Republic of Serbia, Faculty of Biology, University of Belgrade, Institution for Protection of Nature of the Republic of Serbia, Belgrade.

Stojković, Z. (2012): Osredak-močvarno stanište na obalama Zapadne Morave. Zaštita prirode 62(2): 65-81. [in Serbian]

Thaisz, L. de (1907): Additamenta nova Florae Hungaricae. - Magyar Botanikai Lapok 6(5-7): 166-169. [in Hungarian]

Thiers, B. (2019+): Index Herbariorum: A global directory of public herbaria and associated staff. In: New York Botanical Garden's Virtual Herbarium. : 57-61. [http://sweetgum.nybg.org/science/ih/] (accessed: September $\left.4^{\text {th }} 2020\right)$.

Tomović, G., Niketić, M., Ranđelović, V., Stevanović, V. (2005): The vascular flora of Mountain Sokolovica in Central Serbia (Serbia and Montenegro). Flora Mediterranea 15: 9-55.

Turland, N. J., Wiersema, J. H., Barrie, F. R., Greuter, W., Hawksworth, D. L., Herendeen, P. S., Knapp, S., Kusber, W-H., Li, D-Z., Marhold, K., May, T. W., McNeill, J., Monro, A. M., Prado, J., Price, M. J., Smith, G. F. (2018): International Code of Nomenclature for algae, fungi, and plants (Shenzhen Code) adopted by the Nineteenth International Botanical Congress Shenzhen, China, July 2017. - Regnum Vegetabile 159.

Zlatković, B., Ranđelović, V., Amidžić, L. (2005): Flora i vegetacija slatina centralne i južne Srbije i njihova valorizacija sa aspekta zaštite. - Zavod za zaštitu prirode Srbije, Niš. [in Serbian]

Zlatković, I., Zlatković, B., Ranđelović, V., Jenačković, D., Amidžić, L. (2014): Taxonomical, phytogeographical and ecological analysis of the salt marsh flora of Central and Southern Serbia. - Biologica Nyssana 5(2): 91-102.

Zorkóczy, L. (1896): Ujvidék és környékének flórája. - Popovits M. Testvérek könyvnyomdája, Ujvidéken. [in Hungarian] 


\title{
МАТЕРИЈАЛИ ЗА ФЛОРУ СРБИЈЕ ИЗ ХЕРБАРИЈУМСКЕ КОЛЕКЦИЈЕ ПЗЗП (3)
}

\author{
РАНКО ПЕРИЋ, ЈЕЛЕНА КНЕЖЕВИЋ
}

\section{Р Е 3 И М Е}

Чланак садржи трећи део података о пробраним, новим и у флори Србије пажње вреднијим таксонима добијених током ревизије Хербаријумске колекције Покрајинског завода за заштиту природе (PZZP). Одабрани подаци укључују 11 врста, три подврсте, три варијетета, један инфраспецијски таксон са неодређеним статусом [stat. indet.] и једну нотоврсту сврстане у седам родова (Filago L., Ludwigia L., Luzula DC., Lycopus L., Lythrum L., Phlomis L. и Silene L.). Два варијетета (Luzula campestris subsp. campestris var. elata, Silene floscuculi subsp. flos-cuculi var. latifolia) и један инфраспецијски таксон са неодређеним статусом (Luzula multiflora subsp. multiflora [stat. indet.] b. uliginosa) су нови за флору Србије. Поред тога, једна врста (Ludwigia palustris) и једна нотоврста (Lythrum $\times$ scabrum) су потврђене за Србију. 